Una M. Röhr-Sendlmeier, Sebastian Bergold, Andreas Jöris, Anna Verena Cummings, Karin Heim \& Eva Johannen

\title{
Berufstätige Mütter und sozial-emotionale Kompetenzen ihrer Kinder
}

\section{Working mothers and social-emotional competencies of their children}

\section{Zusammenfassung:}

Bei 985 vollständigen Familien mit Kindern der 3., 4. und 6. Klassen aus Grundschulen, Hauptschulen, Realschulen und Gymnasien wurde in einer Querschnittsuntersuchung die Beziehung zwischen der Berufstätigkeit der Mutter und sozial-emotionalen Kompetenzen der Kinder untersucht. Die Aufgabenübernahme des Vaters (erfragt vom Vater), das Wohlbefinden und der praktizierte Erziehungsstil der Mutter (erfragt von der Mutter) wurden als vermittelnde Variablen für den postulierten Zusammenhang angenommen. In Mediatoranalysen der Fragebogendaten wurde der Zusammenhang zwischen Berufstätigkeit und seelischer Gesundheit der Mutter durch die väterliche Aufgabenbeteiligung vermittelt. Für den Zusammenhang zwischen mütterlichem Wohlbefinden und den von den Müttern eingeschätzten sozialemotionalen Kompetenzen der Kinder erwies sich ein autoritativer Erziehungsstil als partieller Mediator. Zwischen dem Umfang der mütterlichen Berufstätigkeit und sozial-emotionalen Kompetenzen der Kinder konnten keine direkten Zusammenhänge gefunden werden, von Bedeutung war jedoch die Kongruenz zwischen gewünschtem und tatsächlichem beruflichem Status der Mutter.

Schlagwörter: mütterliche Berufstätigkeit, Aufgabenbeteiligung, Wohlbefinden, Erziehungsstil, sozial-emotionale Kompetenzen

\begin{abstract}
:
A cross-sectional questionnaire survey of a total of 985 families (parents and children) of 3rd, 4th and 6th graders at different levels in the tripartite German school system was conducted to gain insight into socialization variables connected with maternal employment as to their impact on pupils' social-emotional competencies. Fathers' participation in household and educational tasks (answered by fathers), mothers' well-being and mode of parenting (both answered by mothers) were postulated to mediate between mothers' employment and social-emotional competencies of the children. Mediation analyses showed that fathers' participation mediated the correlation between mothers' employment and mental health. Authoritative parenting partially mediated the correlation between mothers' well-being and children's social-emotional competencies as seen by their mothers. No direct correlation was found between maternal employment and social-emotional competencies. Statistical path analyses revealed that congruence of desired and practiced working status was more relevant for mothers' well-being and children's social-emotional competencies than the amount of hours mothers worked.
\end{abstract}

Key words: working mothers, fathers' participation, psychological well-being, parenting, socialemotional competencies 


\section{Mütterliche Berufstätigkeit und sozial-emotionale Kompetenzen der Kinder - Stand der Forschung}

Bis heute hat die Debatte um die Berufstätigkeit von Müttern nichts von ihrer Aktualität verloren. Besonders in Westdeutschland stoßen berufstätige Mütter immer noch häufig auf Skepsis oder müssen sich gar Vorwürfe gefallen lassen, sie vernachlässigten durch ihre beruflichen Aktivitäten ihr Kind, was negative Folgen für dessen sozial-emotionale und kognitive Entwicklung nach sich ziehe. Die vorliegende Studie setzt sich daher mit den Zusammenhängen zwischen mütterlicher Berufstätigkeit und sozial-emotionalen Kompetenzen der Kinder auseinander, wobei sie entgegen der landläufigen Meinung und in Einklang mit neueren Studienergebnissen annimmt, dass eine Erwerbstätigkeit der Mutter eine positive Einflussgröße für die sozial-emotionale Entwicklung des Kindes darstellt. Als vermittelnde Variablen für diesen Zusammenhang werden eine vermehrte Aufgabenübernahme des Vaters im Haushalt und bei der Kindererziehung, das Wohlbefinden sowie der Erziehungsstil der Mutter angenommen.

Die Quote berufstätiger Frauen und Mütter steigt bis heute stetig an: Den Angaben des Statistischen Bundesamts (2011) zufolge waren im Jahr 2009 72\% der 25- bis 49-jährigen Mütter mit mindestens einem im Haushalt lebenden Kind unter 25 Jahren erwerbstätig. Untersuchungen setzen sich zunehmend mit den Auswirkungen mütterlicher Berufstätigkeit auf die Entwicklung der Kinder auseinander. Bis in die 1960er und frühen 1970er Jahre hinein wurde die Berufstätigkeit der Mutter aufgrund des großen Einflusses der Bindungstheorie als schädlich für das Kind betrachtet. Bereits 1979 stellte jedoch Ursula Lehr fest, dass die mütterliche Berufstätigkeit weder positive noch negative Auswirkungen auf die Entwicklung des Kindes habe. Armistead/Wierson/Forehand (1990) konnten in ihrer Querschnittstudie mit 145 Jugendlichen im Alter zwischen 11;5 und 14;10 Jahren ebenfalls keine negativen Effekte bezüglich kognitiver Fähigkeiten, sozialer Kompetenzen, Verhaltensproblemen und der Mutter-Kind-Beziehung nachweisen.

In neueren Publikationen vor allem aus dem anglo-amerikanischen Raum wird die Berufstätigkeit der Mutter sogar als positive Einflussgröße gesehen. So fanden Hoffman/ Youngblade (1999) in ihrer Querschnittstudie mit Drittklässlern, dass Kinder - insbesondere Töchter - berufstätiger Mütter neben besseren Schulleistungen außerdem ein besser entwickeltes Sozialverhalten aufwiesen, wobei die vermittelnden Variablen ein stärkeres Wohlbefinden der Mutter, ein autoritativerer Erziehungsstil und die Beteiligung des Vaters an Haushalts- und Kinderbetreuungsaufgaben waren. Sei die Mutter berufstätig, übernehme der Vater mehr Aufgaben, was ein höheres Wohlbefinden der Mutter durch eine Entlastung von ihrer Doppelrolle nach sich ziehe, was sich wiederum in ihrem Erziehungsverhalten und in den sozial-emotionalen Kompetenzen der Kinder niederschlage. In ihrer Längsschnittstudie mit knapp 12.000 Kindern und drei Erhebungszeitpunkten, als die Kinder ein Jahr, drei Jahre und fünf Jahre alt waren, konnten McMunn/Kelly/Cable/ Bartley (2011) zeigen, dass Kinder, deren Mütter kurz nach der Geburt in die Erwerbstätigkeit zurückgekehrt waren, weniger Verhaltens- und Sozialprobleme aufwiesen als Kinder von nichtberufstätigen Müttern. Die Effekte fielen für Mädchen etwas stärker aus, waren jedoch für beide Geschlechter signifikant. Es profitierten diejenigen Kinder am meisten, deren Eltern beide berufstätig waren, aber den Beruf dennoch gut mit dem Familienleben vereinbaren konnten. Die Autoren vermuten, dass diese Eltern die höchste $\mathrm{Zu}$ - 
friedenheit und Ausgeglichenheit besäßen, was sich positiv auf die sozial-emotionale Entwicklung der Kinder auswirke. In einer Meta-Analyse mit 69 Studien und insgesamt fast 129.000 Kindern zu den Auswirkungen mütterlicher Berufstätigkeit im Säuglingsund Kleinkindalter auf spätere sozial-emotionale Kompetenzen und Schulleistungen zeigten sich positive Effekte der Berufstätigkeit im Lehrerurteil (Lucas-Thompson/Goldberg/ Prause 2010). Die Autoren stellten allerdings auch fest, dass sich ansonsten keine Zusammenhänge zeigten. Wurden Moderatorvariablen beachtet, ergaben sich differenziertere Ergebnisse: So zeigte sich, dass die Berufstätigkeit der Mutter eher positive Effekte hatte, wenn die Mutter alleinerziehend war (ein Ergebnis, das auch der deutsche Kinder- und Jugendgesundheitssurvey des Robert-Koch-Instituts bestätigen konnte, vgl. Hölling/ Schlack 2008), wenn die Familie über einen niedrigen sozioökonomischen Status verfügte und wenn das Kind bei Wiederaufnahme der Berufstätigkeit mindestens zwei Jahre alt war. Eher negative Effekte traten auf, wenn die Mutter nicht alleinerziehend war, wenn die Familie mindestens der Mittelschicht angehörte und wenn das Kind bei Wiederaufnahme der Berufstätigkeit höchstens ein Jahr alt war (s. auch Goldberg/Prause/LucasThompson/Himsel 2008). Insgesamt waren die Effektstärken mit Beträgen zwischen $r=$ .015 und $r=.137$ (positive Effekte) bzw. höchstens $r=.035$ (negative Effekte) jedoch klein. In einer aktuellen deutschen Längsschnittstudie von Jaursch/Lösel (2011), die ihren Fokus auf kindliche Erlebens- und Verhaltensprobleme in Abhängigkeit von der mütterlichen Berufstätigkeit legt und in der 660 Kinder im Vorschulalter über sechs Jahre hinweg untersucht wurden, konnten keine negativen Effekte auf die Kinder durch eine Erwerbstätigkeit der Mutter in den ersten drei Lebensjahren des Kindes (retrospektiv erfragt) oder durch eine aktuelle Berufstätigkeit zum jeweiligen Erhebungszeitpunkt festgestellt werden.

Interessant ist die Sicht der Kinder auf die Berufstätigkeit der Mutter: Nach dem in Deutschland durchgeführten „GEOlino-Kinderwertemonitor 2010“ (GEOlino/Kinderhilfswerk der Vereinten Nationen 2010) betrachten die Kinder die Berufstätigkeit beider Eltern inzwischen als selbstverständlich. Ca. 90\% der repräsentativ ausgewählten 1.500 6bis 14-jährigen Kinder gaben an, froh bzw. stolz zu sein, dass ihre Mutter einer Arbeit nachgehe; $80 \%$ fanden, dass ihre Mutter trotz Berufstätigkeit unter der Woche genug Zeit für sie habe. Als besondere Pluspunkte führten sie an, dass sie mehr Freiheiten hätten, die Mutter zufriedener sei und mehr soziale Kontakte durch die Berufstätigkeit entstünden. Diese Daten werden auch durch die 2. World-Vision-Kinderstudie (Hurrelmann/Andresen 2010) gestützt: Nur ca. 8\% der Kinder beklagten sich über zu wenig Zeit mit den Eltern, wenn ein Elternteil Vollzeit und das andere Teilzeit erwerbstätig war. Bei zwei Vollzeit erwerbstätigen Elternteilen stieg diese Zahl auf 18\%, bei arbeitslosen Eltern aber auf ca. $31 \%$. Abramovitch/Johnson stellten in ihrer Untersuchung mit Dritt- und Viertklässlern bereits 1992 fest, dass die Kinder ihre Mütter als zufriedener wahrnahmen, wenn diese einem Beruf nachgingen. Der Zufriedenheit der Kinder war die Erwerbstätigkeit der Mutter nicht abträglich, solange diese keine Schichtarbeit mit Spät- oder Nachtdiensten leistete. 


\subsection{Berufstätigkeit und Wohlbefinden der Mutter}

Berufstätigkeit wird als eine wesentliche Determinante des psychischen Wohlbefindens und der Lebenszufriedenheit betrachtet, da neben der manifesten Funktion der wirtschaftlichen Existenzsicherung auch noch zahlreiche latente Funktionen erfüllt werden (Jahoda 1981): Die Strukturierung der Tages- und Lebenszeit, Erreichung von Selbsttranszendenz, Status- und Identitätsfindung, psychophysische Aktivierung sowie die Pflege sozialer Kontakte außerhalb der Familie. Frauen sehen ihre Berufstätigkeit sogar sehr viel stärker als Männer als sinnstiftend (Fthenakis 1999), und für ihr Selbstbewusstsein spielt der Beruf nach aktuellen Befragungsergebnissen eine zentrale Rolle (Bundesministerium für Familie, Senioren, Frauen und Jugend 2010). Diese Befunde stehen in Einklang mit der Bereicherungshypothese (Gove 1984), nach der die simultane Ausübung mehrerer Rollen zusätzliche Belohnungs- und Kompensationsmöglichkeiten bietet, berufstätigen Müttern also einen Zugewinn an Selbstwertgefühl, finanziellen Ressourcen, sozialen Kontakten und Erfolgserlebnissen und damit eine bessere physische sowie psychische Gesundheit beschert. Tatsächlich zeigen berufstätige Mütter in vielen einschlägigen Studien einen besseren Gesundheitszustand und ein höheres subjektives Wohlbefinden als nichtberufstätige Mütter (Repetti/Matthews/Waldron 1989; Hoffman/Youngblade 1999; Klumb/Lampert 2004; Richter/Bergmann/Bergmann/Dudenhausen 2007; Buehler/O'Brien 2011). Längsschnittstudien zum Zusammenhang zwischen Berufstätigkeit und Wohlbefinden legen die Vermutung nahe, dass die kausale Wirkung in beide Richtungen verlaufen kann, meist jedoch die Berufstätigkeit auf das Wohlbefinden einwirkt (Wethington/Kessler 1989; Waldron/Weiss/Hughes 1998; Paul/Moser 2001; Raver 2003; Klumb/Lampert 2004). Querschnittliche Untersuchungen in Deutschland ermittelten jedoch auch vergleichbare Lebenszufriedenheiten bei berufstätigen und nichtberufstätigen Müttern, allerdings systematische Unterschiede in der Leistungsmotivation und der aktiven Freizeitgestaltung mit dem Kind zugunsten der Berufstätigen (Röhr-Sendlmeier 2009; MüllerGünther 2010; Röhr-Sendlmeier/Kröger 2011). Weitere Studien weisen auf die Bedeutung der Kongruenz zwischen dem erwünschten und dem realisierten Ausmaß der Berufstätigkeit für die Lebenszufriedenheit der Mütter hin (Berger 2009; Berger 2011).

Neben der Berufstätigkeit an sich wird auch die durch die Erwerbstätigkeit der Partnerin erhöhte Teilnahme des Vaters an Haushalts- und Kindererziehungsaufgaben als positive Einflussgröße auf das Wohlbefinden der Mutter angenommen, da die Mutter von ihrer Doppelrolle entlastet werde. In der Tat konnten sowohl in Quer- als auch in Längsschnittstudien empirisch positive Zusammenhänge zwischen dem Umfang der mütterlichen Berufstätigkeit und der Partizipation des Vaters einerseits (Crouter/Crowley 1990; Almeida/Maggs/Galambos 1993; Hoffman/Youngblade 1999; Pache 2009; Berger 2011; Röhr-Sendlmeier/Bergold 2012) sowie zwischen der väterlichen Partizipation und dem Wohlbefinden der Mutter andererseits aufgewiesen werden (Schwartzberg/Dytell 1996; Hoffman/Youngblade 1999; Wiese 2004; Berger 2011). 


\subsection{Berufstätigkeit und Erziehungsstil der Mutter}

Verschiedene Studien konnten einen Zusammenhang zwischen der Berufstätigkeit der Mutter und dem praktizierten Erziehungsstil belegen. Hoffman/Youngblade (1999) fanden heraus, dass nichtberufstätige Mütter einen permissiveren oder einen autoritäreren Erziehungsstil anwendeten als berufstätige Mütter. Diese erzogen eher autoritativ, d.h., sie erwarteten die Einhaltung von Regeln, berücksichtigten dabei aber auch die Belange des Kindes. Nichtberufstätige Mütter legten unabhängig vom sozialen Status mehr Wert auf Gehorsam und autoritäre Verhaltenskontrolle als berufstätige Mütter. Gottfried/Gottfried (2006) konnten, diesen Befund untermauernd, zeigen, dass berufstätige Mütter die Selbständigkeit ihrer Kinder früher förderten als nichtberufstätige Mütter. In einer Studie von Raver (2003) mit zwei Erhebungszeitpunkten über einen Zeitraum von zwei Jahren (das Alter der Kinder zu t $t_{1}$ betrug zwischen 3.8 und 4.6 Jahre) zog ein gestiegener Umfang der mütterlichen Berufstätigkeit ebenfalls positive Folgen für die Kindererziehung nach sich: Nahm der Umfang der Berufstätigkeit von $\mathrm{t}_{1} \mathrm{zu} \mathrm{t}_{2} \mathrm{zu}$, drückten Mütter zu $\mathrm{t}_{2}$ weniger Ärger gegenüber ihren Kindern aus, waren emotional ausgeglichener bei der Erledigung von Kinderbetreuungsaufgaben und setzten weniger auf Bestrafung. Nach Hoffman (1989) werden Kinder aus Familien, in denen beide Elternteile erwerbstätig sind, früher zu Unabhängigkeit und Selbständigkeit angehalten, da so die alltäglichen Anforderungen des Familienlebens besser bewältigt werden könnten. Zudem empfänden Mütter, die sich ausschließlich für die Betreuung und Erziehung des Kindes zuständig sehen, die aufkommende Selbständigkeit ihrer Kinder möglicherweise als Bedrohung ihrer eigenen Rolle und ihres Selbstwertes.

Neben dem Wert, der einer frühen Selbständigkeit des Kindes zugesprochen wird, scheint jedoch auch das Wohlbefinden der Mutter, das positiv mit ihrer Berufstätigkeit zusammenhängt, günstig auf den Erziehungsstil zu wirken. Bei Hoffman und Youngblade (1999) hingen Depressivitätswerte der Mütter mit einem permissiveren und autoritäreren, die Lebenszufriedenheit mit einem autoritativeren Erziehungsstil zusammen. Rutter (1990) führt aus, dass Mütter mit höheren Depressivitätswerten Bedürfnisse und Signale ihrer Kinder weniger wahrnehmen und auf sie eingehen, sich weniger unterstützend verhalten, weniger positive und mehr negative Emotionen gegenüber ihrem Kind zeigen und in ihrem Erziehungsverhalten weniger konsistent sind als Mütter mit niedrigeren Depressivitätswerten.

\subsection{Wohlbefinden und Erziehungsstil der Mutter und sozial-emotionale Kompetenzen der Kinder}

Empirische Ergebnisse legen nahe, dass elterliche Zufriedenheit und elterlicher Erziehungsstil mit sozial-emotionalen Kompetenzen der Kinder in Verbindung stehen, so dass beide Variablen als Mediatoren für den Zusammenhang zwischen mütterlicher Berufstätigkeit und sozial-emotionalen Kompetenzen der Kinder angenommen werden können. Ein höheres Wohlbefinden der Mütter aus der „Arbeiterschicht“ in der Studie von Hoffman/Youngblade (1999) hing zusammen mit einer höheren Frustrationstoleranz, weniger ausagierendem Verhalten, höherer Beliebtheit bei den Mitschülern und weniger phy- 
sischer und verbaler Aggressivität bei ihren Söhnen. In der Mittelschicht hatten Töchter zufriedenerer Mütter internalere Kontrollüberzeugungen sowie eine geringere Schüchternheit und Ängstlichkeit als Töchter von weniger zufriedenen Müttern. In einer Untersuchung mit 291 Schülerinnen und Schülern aus bildungsfernen Familien zeigten die Kinder ein umso positiveres schulisches Selbstkonzept, je zufriedener die Mütter mit der Unterstützung bei der Kindererziehung und -betreuung durch Dritte waren (Schnittert 2011). Der Zusammenhang zwischen elterlichem Erziehungsstil und der sozial-emotionalen Entwicklung der Kinder gilt als gut belegt. So fördert der autoritative Erziehungsstil durch einen einfühlsamen, unterstützenden und demokratischen Umgang mit den Kindern etwa die Ausbildung von positiven Selbstkonzepten, Selbstwirksamkeitsüberzeugungen, Empathie, prosozialem Verhalten und Beliebtheit bei Gleichaltrigen, während der autoritäre bzw. der permissive Erziehungsstil zur Entwicklung von externalen Kontrollüberzeugungen, Schüchternheit, Ängsten oder aggressiven Verhaltensweisen beitragen kann (z.B. Deković/Janssens 1992; Hoffman/Youngblade 1999; Franiek/Reichle 2007; Reichle/Gloger-Tippelt 2007). Hoffman/Youngblade (1999) gehen auf der Basis von Mediatoranalysen davon aus, dass sich ein hohes Wohlbefinden in einem autoritativeren Erziehungsstil niederschlage, welcher sich wiederum förderlich auf die sozial-emotionale Entwicklung der Kinder auswirke.

\section{Hypothesen}

Auf Basis der im vorangegangenen Abschnitt erläuterten Forschungsbefunde wurde ein Modell entwickelt (siehe Abbildung 1), in dem Wirkzusammenhänge zwischen mütterlicher Berufstätigkeit, väterlicher Beteiligung an Haushalts- und Kindererziehungsaufgaben, Wohlbefinden der Mutter, Erziehungsstil sowie den sozial-emotionalen Kompetenzen des Kindes postuliert wurden. Im Einzelnen wurden folgende Hypothesen aufgestellt:

Hypothese 1: Die Aufgabenpartizipation des Vaters mediiert zwischen der Berufstätigkeit und dem Wohlbefinden der Mutter.

Als Voraussetzung für eine mögliche Mediation waren folgende Einzelhypothesen zu überprüfen:

- Je umfangreicher die Berufstätigkeit der Mutter, desto höher ist ihr Wohlbefinden (1.1).

- Je umfangreicher die Berufstätigkeit der Mutter, desto ausgeprägter ist die Aufgabenpartizipation des Vaters (1.2).

- Je ausgeprägter die Aufgabenpartizipation des Vaters, desto höher ist das Wohlbefinden der Mutter (1.3).

Hypothese 2: Der Erziehungsstil der Mutter mediiert zwischen dem Wohlbefinden der Mutter und den sozial-emotionalen Kompetenzen des Kindes.

Als Voraussetzung für eine mögliche Mediation waren folgende Einzelhypothesen zu überprüfen: 
- Je höher das Wohlbefinden der Mutter, desto ausgeprägter sind die sozialemotionalen Kompetenzen des Kindes (2.1).

- Je höher das Wohlbefinden der Mutter, desto autoritativer und desto weniger permissiv und autoritär ist ihr Erziehungsstil (2.2).

- Je positiver der Erziehungsstil (autoritativ, wenig permissiv und wenig autoritär) der Mutter, desto ausgeprägter sind die sozial-emotionalen Kompetenzen des Kindes (2.3).

Hypothese 3: Der Erziehungsstil der Mutter mediiert zwischen dem Umfang der Berufstätigkeit der Mutter und den sozial-emotionalen Kompetenzen des Kindes.

Als Voraussetzung für eine mögliche Mediation waren zusätzlich zu Hypothese 2.3 folgende Einzelhypothesen zu überprüfen:

- Je umfangreicher die Berufstätigkeit der Mutter, desto ausgeprägter sind die sozialemotionalen Kompetenzen des Kindes (3.1).

- Je umfangreicher die Berufstätigkeit der Mutter, desto autoritativer und desto weniger permissiv und autoritär ist ihr Erziehungsstil (3.2).

Hypothese 4: Das Wohlbefinden der Mutter mediiert zwischen dem Umfang der Berufstätigkeit der Mutter und den sozial-emotionalen Kompetenzen des Kindes.

Voraussetzung für eine mögliche Mediation war die Bestätigung der Hypothesen 1.1, 2.1 und 3.1.

Abbildung 1: Den Hypothesen zugrunde liegendes Gesamtmodell

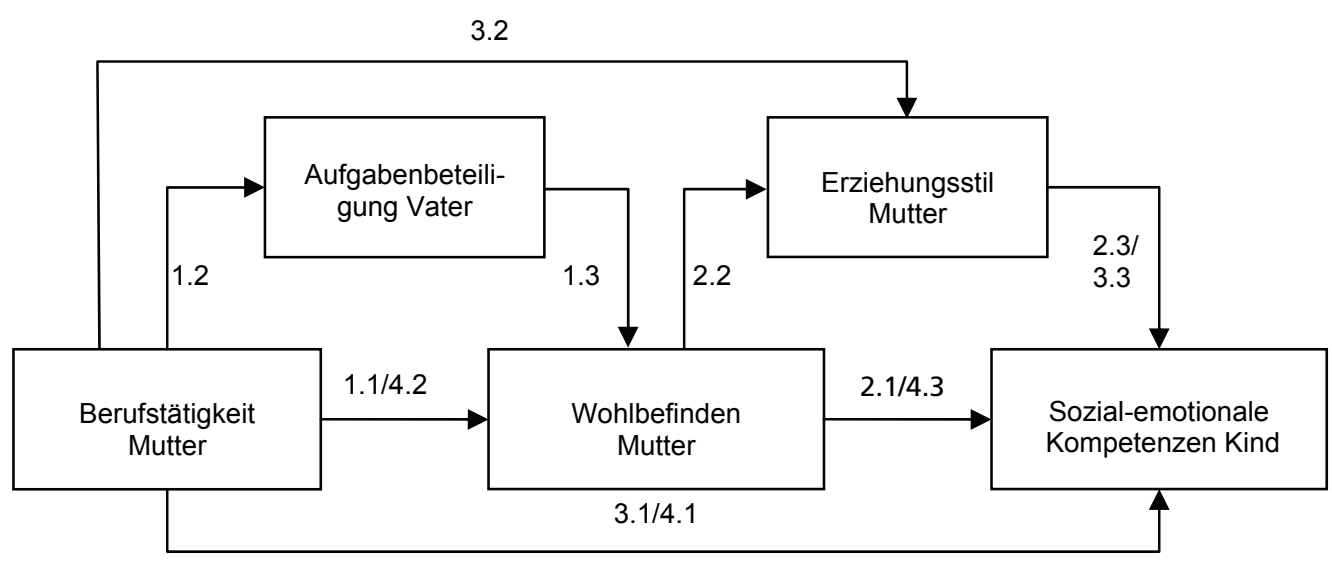




\section{Methode}

\subsection{Design und Stichprobe}

Bei der vorliegenden Untersuchung handelt es sich um eine fragebogenbasierte Ex-postfacto-Studie. In 12 Grundschulen, 7 Hauptschulen, 2 Realschulen und 3 Gymnasien aus ländlichen und suburbanen Bezirken in Nordrhein-Westfalen sowie aus urbanen Gebieten Baden-Württembergs wurden Kinder und ihre (Stief-)Eltern klassenweise kontaktiert. Von 1.170 Familien wurden ausgefüllte Bögen zurückgegeben, was einer Rücklaufquote von $61.5 \%$ entspricht. 185 Bögen wurden ausgeschlossen, weil sie von allein erziehenden Eltern stammten oder nicht beide Elternbögen vorlagen, so dass die endgültige Stichprobe 985 vollständige Familien umfasst (250 Familien mit Drittklässlern, 255 Familien mit Viertklässlern, 480 Familien mit Sechstklässlern; davon 505 Grundschüler, 145 Hauptschüler, 107 Realschüler, 228 Gymnasiasten). Die Gesamtstichprobe umfasst 2.955 Personen. Nach Bundesländern (und damit unterschiedlichem Urbanisierungsgrad) gesonderte Datenanalysen offenbarten keine überzufälligen Unterschiede in den Ergebnissen, so dass beide Stichproben zusammengefasst wurden.

$51.4 \%(n=506)$ der Schüler waren Mädchen, 48.6\% $(n=479)$ Jungen. Ihr Alter betrug im Schnitt 10.80 Jahre $(S D=1.38) .94 .4 \%(n=930)$ der Eltern waren verheiratet; $5.6 \%(n=55)$ lebten in einer festen Partnerschaft. Tabelle 1 informiert über Bildungsabschlüsse und Umfang der Berufstätigkeit der befragten Mütter und Väter.

Tabelle 1: Höchster Bildungsabschluss und Umfang der Berufstätigkeit bei den untersuchten Müttern und Vätern

\begin{tabular}{lcc}
\hline & Mütter & Väter \\
\hline Höchster Bildungsabschluss & & \\
kein (Fach-)Abitur & $70.4 \%(n=693)$ & $58.6 \%(n=577)$ \\
(Fach-)Abitur & $14.0 \%(n=138)$ & $10.1 \%(n=100)$ \\
(Fach-)Hochschulstudium & $14.3 \%(n=141)$ & $26.7 \%(n=263)$ \\
k.A. & $1.3 \%(n=13)$ & $4.6 \%(n=45)$ \\
Umfang Berufstätigkeit & & \\
nicht berufstätig & $29.3 \%(n=289)$ & $2.8 \%(n=27)$ \\
Teilzeit (ca. 25\%) & $35.0 \%(n=345)$ & $0.9 \%(n=9)$ \\
halbtags & $15.7 \%(n=154)$ & $0.4 \%(n=4)$ \\
Teilzeit (ca. 75\%) & $12.1 \%(n=119)$ & $1.4 \%(n=14)$ \\
Vollzeit & $7.4 \%(n=73)$ & $93.9 \%(n=925)$ \\
k.A. & $0.5 \%(n=5)$ & $0.6 \%(n=6)$ \\
\hline
\end{tabular}

Anmerkung: k.A. $=$ keine Angabe.

\subsection{Instrumente}

- Berufstätigkeit und soziodemographische Merkmale. Neben Alter, Geschlecht und Klasse des Kindes sowie dem Familienstand der Eltern wurden der höchste Bildungsabschluss von Mutter und Vater, der aktuell ausgeübte Beruf und der Umfang der Berufstätigkeit (Arbeitsstunden pro Woche) erfasst. Zudem wurde in Anlehnung an Wethington/ 
Kessler (1989) die Kongruenz zwischen gewünschtem und tatsächlichem Beschäftigungsstatus erhoben (,,Wenn Sie die Wahl hätten, würden Sie lieber...? Teilzeit arbeiten, Vollzeit arbeiten, nicht arbeiten").

- Die relative Aufgabenpartizipation des Vaters im Haushalt und bei kindbezogenen Aufgaben wurde mithilfe einer modifizierten Form der ,Who Does It More? “ Measure aus Hoffman/Youngblade (1999) erfasst, die - wie die weiteren ursprünglich amerikanischen Skalen (s.u.) - ins Deutsche übersetzt und durch Rückübersetzung ins Englische validiert wurde (hierzu auch Röhr-Sendlmeier/Schäfer/Vonderbank 2009). Mit 29 Items wurden die Väter hinsichtlich der Verantwortlichkeit für die Erledigung verschiedener Tätigkeiten im Haushalt und bei der Kindererziehung bzw. -betreuung auf einer 5-stufigen Ratingskala befragt ( $1=$,Mutter", 2 = ,Meist die Mutter, manchmal der Vater", 3 = „Beide etwa gleich “, 4 = „Meist der Vater, manchmal die Mutter“", 5 = „Vater"). In explorativen Hauptkomponentenanalysen mit Varimax-Rotation ergaben sich die beiden Dimensionen „Haushalt und funktionale Kindererziehung“ (z.B. „Wäsche waschen“, „Mit den Kindern zum Arzt gehen“; $\alpha=.859$ ) und „zielgerichtete Kindererziehung“ (z.B. „Den Kindern Sachen erklären/Fragen beantworten“, „Ihnen bei den Hausaufgaben helfen"; $\alpha$ $=.795)$. Für das gesamte Instrument ergab sich eine interne Konsistenz von $\alpha=.888$.

- Das subjektive Wohlbefinden der Mutter wurde über Selbstauskünfte in drei Skalen operationalisiert: Die allgemeine Lebenszufriedenheit (z.B. „Mein Leben könnte kaum glücklicher sein, als es ist") und das Stimmungsniveau (z.B. „Ich fühle mich meist ziemlich fröhlich") wurden mit den entsprechenden Subskalen der Habituellen Subjektiven Wohlbefindensskala (HSWBS) von Dalbert (1992) erfasst. Im Vergleich zur Originalversion wurden die Skalen leicht modifiziert: Das Antwortformat wurde von 6 auf 5 Stufen reduziert und die Pole verbal verankert $(1=$, ,stimmt nicht", $5=$,stimmt sehr" $)$. In Faktorenanalysen luden zwei Items der Stimmungsniveau-Skala höher auf der Lebenszufriedenheitsskala, so dass die ursprünglichen Itemanzahlen (Lebenszufriedenheit: 7 Items; Stimmungsniveau: 6 Items) nun 9 bzw. 4 betrugen. Cronbachs $\alpha$ lag bei .921 (Lebenszufriedenheit) und .793 (Stimmungsniveau). Zusätzlich wurden 8 nach inhaltlicher Relevanz und Trennschärfe ausgewählte Items der Skala Seelische Gesundheit des Trierer Persönlichkeitsfragebogens (TPF) (Becker 1989) eingesetzt (z.B. „Ich fühle mich dem Leben und seinen Schwierigkeiten eigentlich ... gut gewachsen"), deren 4-stufiges Antwortformat auf 5 Stufen erweitert wurde $\left(1=\right.$, ,immer", $5=$ „nie $\left.e^{\text {" }}\right)(\alpha=.811)$. Ergänzend wurde die Lebenszufriedenheit des Vaters mit der entsprechenden Skala aus der HSWBS erfasst (7 Items; $\alpha=.893$ ).

- Der mütterliche Erziehungsstil wurde mithilfe von 15 Items nach Hoffman/Youngblade (1999) erhoben, die die Erziehungsstile nach Baumrind (1966) erfassen. Das Antwortformat wurde von einer 6-stufigen auf eine 5-stufige Skala reduziert $(1=$, ,stimmt nicht", $5=$,stimmt sehr"). Die Originalskala enthält 20 Items, aufgrund geringer Trennschärfen wurden jedoch 5 Items ausgeschlossen. Der autoritative Erziehungsstil wurde somit mit 6 Items (z.B. „Kinder sollen sich an Familienentscheidungen beteiligen dürfen“; $\alpha=.636$ ), der autoritäre Erziehungsstil mit 5 Items (z.B. „Es ist sehr wichtig, dass Kinder Autoritäten respektieren"; $\alpha=.660$ ) und der permissive Erziehungsstil mit 4 Items (z.B. ,Je weniger Regeln und Disziplin Kinder bekommen, desto besser ist es für sie“; $\alpha=$ .510) erfasst. Die Hauptkomponentenanalyse bestätigte die erwartete Faktorenstruktur. 
Die internen Konsistenzen der Skalen blieben hinter den Erwartungen zurück, wurden jedoch als für Forschungszwecke noch ausreichend bewertet.

- Zur Erfassung der sozial-emotionalen Kompetenzen der Kinder wurden sowohl die Mütter als auch die Kinder selbst als Datenquellen herangezogen. Die Müttereinschätzungen der sozialen Kompetenzen erfolgten über zwei Subskalen der Home \& Community Social Behavior Scales (HCSBS) (Merrell/Caldarella 2008), bei denen auf einer 5-stufigen Ratingskala angegeben wird, wie oft das Kind in den letzten drei Monaten die jeweils genannte Verhaltensweise gezeigt hat ( $1=$,nie“, $5=$,häufig"). Die Skala Peer Relations thematisiert Verhaltensweisen, die relevant sind für das Schließen von Freundschaften, die Zugehörigkeit zu einer Gruppe und die Beliebtheit bei Gleichaltrigen (z.B. „Bietet Gleichaltrigen Hilfe an, wenn diese benötigt wird“, „Fordert Gleichaltrige auf, an Aktivitäten teilzunehmen"). Die 10 auf Basis inhaltlicher Relevanz und Trennschärfe ausgewählten Items wiesen eine interne Konsistenz von $\alpha=.876$ auf. Die Skala Self-Management/Compliance erfragt Verhaltensweisen, die sich auf die Erfüllung von Erwartungen Erwachsener beziehen und die Fähigkeit zur Selbstkontrolle anzeigen (z.B. „Hört Anweisungen von Eltern oder Aufsichtspersonen an und führt sie aus", ,Beherrscht sich, wenn er/sie ärgerlich ist"). Hier lag die interne Konsistenz der 9 ausgewählten Items bei $\alpha$ $=.849$.

Selbstkonzept und Empathiefähigkeit der Kinder wurden über Selbstauskünfte erfasst. Das Selbstkonzept wurde mithilfe der deutschen Fassung des Self-Perception-Profile for Children (SPPC-D) (Asendorpf/von Aken 1993) erhoben, bei dem die Beantwortung in zwei Schritten erfolgt. Zunächst beurteilt das Kind, welche von zwei vorgegebenen Aussagen besser auf es zutrifft. Im zweiten Schritt beurteilt es, ob die gewählte Aussage genau oder nur ungefähr zutrifft. Ausgewählt wurden die aus jeweils 6 Items bestehenden Skalen Kognitive Kompetenz (z.B. „Einige Kinder glauben, dass sie sehr gut in ihren Hausaufgaben sind“ vs. „Andere Kinder machen sich Sorgen, ob sie ihre Hausaufgaben richtig machen“; $\alpha=.734$ ), Peerakzeptanz (z.B. „Einige Kinder finden es schwer, Freunde zu finden" vs. „Andere Kinder finden es ziemlich leicht, Freunde zu finden") und Globales Selbstwertgefühl (z.B. „Einige Kinder sind oft unglücklich über sich“ vs. „Andere Kinder sind ziemlich zufrieden mit sich"), wobei die beiden letzten Skalen auf Basis eigens durchgeführter Faktorenanalysen zu einem Faktor zusammengefasst wurden ( $\alpha=$ $.817)$.

Die Empathiefähigkeit der Kinder wurde mithilfe der Skala Empathie aus dem Inventar zur Erfassung von Impulsivität, Risikoverhalten und Empathie bei 9- bis 14-jährigen Kindern (IVE) (Stadler/Janke/Schmeck 2004) erhoben. 8 Items wurden nach ihrer Verständlichkeit und ihrer Trennschärfe ausgewählt (z.B. ,Ich habe Mitleid mit einem Kind, das neu in der Klasse ist", „Ich werde traurig, wenn einer meiner Freunde unglücklich ist"; $\alpha=.860$ ). Die Antworten konnten auf einer modifizierten Antwortskala von 1 bis 5 gegeben werden $(1=$, stimmt gar nicht", $5=$,stimmt genau" $)$.

Eine Übersicht über die erhobenen Variablen bietet Tabelle 2. 
Tabelle 2: Überblick über die erhobenen Variablen sowie Mittelwerte und Standardabweichungen der verwendeten Skalen

\begin{tabular}{|c|c|c|c|}
\hline Variable & $M$ & $S D$ & Adressat \\
\hline $\begin{array}{l}\text { Soziodemographische Merkmale } \\
\text { Alter, Geschlecht und Klasse des Kindes } \\
\text { Familienstand } \\
\text { Höchster Bildungsabschluss } \\
\text { Aktuell ausgeübter Beruf } \\
\text { Umfang der Berufstätigkeit } \\
\text { Kongruenz }\end{array}$ & & & Mutter, Vater \\
\hline $\begin{array}{l}\text { Relative Aufgabenteilung } \\
\text { Haushalt und funktionale Kindererziehung } \\
\text { Zielgerichtete Kindererziehung }\end{array}$ & $\begin{array}{l}1.958 \\
2.529\end{array}$ & $\begin{array}{l}.490 \\
.432\end{array}$ & Vater \\
\hline Subjektives Wohlbefinden & & & \\
\hline $\begin{array}{l}\text { Allgemeine Lebenszufriedenheit (Mutter) } \\
\text { Allgemeine Lebenszufriedenheit (Vater) } \\
\text { Stimmungsniveau } \\
\text { Seelische Gesundheit }\end{array}$ & $\begin{array}{l}3.894 \\
3.903 \\
3.407 \\
3.930\end{array}$ & $\begin{array}{l}.702 \\
.661 \\
.786 \\
.432\end{array}$ & $\begin{array}{l}\text { Mutter } \\
\text { Vater } \\
\text { Mutter } \\
\text { Mutter }\end{array}$ \\
\hline $\begin{array}{l}\text { Mütterlicher Erziehungsstil } \\
\text { Autoritativer Stil } \\
\text { Autoritärer Stil } \\
\text { Permissiver Stil }\end{array}$ & $\begin{array}{l}4.078 \\
3.110 \\
2.069\end{array}$ & $\begin{array}{l}.494 \\
.692 \\
.569\end{array}$ & Mutter \\
\hline Sozial-emotionale Kompetenzen des Kindes & & & \\
\hline $\begin{array}{l}\text { Peer Relations } \\
\text { Self-Management/Compliance } \\
\text { Selbstkonzept kognitive Kompetenz } \\
\text { Peerakzeptanz/globales Selbstwertgefühl } \\
\text { Empathiefähigkeit }\end{array}$ & $\begin{array}{l}4.213 \\
3.748 \\
2.933 \\
3.109 \\
3.700\end{array}$ & $\begin{array}{l}.557 \\
.588 \\
.627 \\
.543 \\
.908\end{array}$ & $\begin{array}{l}\text { Mutter } \\
\text { Mutter } \\
\text { Kind } \\
\text { Kind } \\
\text { Kind }\end{array}$ \\
\hline
\end{tabular}

\subsection{Auswertung}

Die Hypothesentestungen wurden in einem ersten Schritt mittels Mediatoranalysen nach Baron/Kenny (1986) vorgenommen. Gemäß dieser muss die Prädiktorvariable zum einen die Mediatorvariable und zum anderen die Kriteriumsvariable signifikant vorhersagen. Wird anschließend eine multiple Regression auf die Kriteriumsvariable mit der Prädiktorund der Mediatorvariable durchgeführt, muss die Mediatorvariable die Kriteriumsvariable trotz Hereinnahme des Prädiktors signifikant vorhersagen, während das $\beta$-Gewicht des Prädiktors durch die Hereinnahme des Mediators abgeschwächt werden muss. Der Grad der Abschwächung entscheidet darüber, ob es sich um eine vollständige oder um eine partielle Mediation handelt. In einem zweiten Schritt wurden die Mediationseffekte mit der Bootstrap-Methode statistisch abgesichert (Preacher/Hayes 2008). In einem dritten Schritt wurden schließlich die vermittelnden Effekte innerhalb des Gesamtmodells auf Signifikanz überprüft (Hayes 2012). Gewählt wurde ein nominelles $\alpha$-Niveau von 0.05. Aufgrund der Vielzahl der Signifikanztestungen wurde zur Kontrolle der Alpha-Fehler-Kumulierung eine family-wise-Adjustierung mit Bonferroni-Korrektur durchgeführt. Fehlende Werte innerhalb einer Skala wurden durch den Skalenmittelwert ersetzt. 


\section{Ergebnisse}

\subsection{Hypothese 1}

Anhand der in Tabelle 3 dargestellten Produkt-Moment-Korrelationen kann festgehalten werden, dass der Umfang, in dem die Mütter berufstätig waren, nur schwach mit den $\mathrm{Zu}-$ friedenheitsvariablen der Mütter zusammenhing. Nur die Korrelation mit der seelischen Gesundheit behielt nach der Bonferroni-Korrektur ihre Signifikanz. Der Umfang der mütterlichen Berufstätigkeit korrelierte dagegen signifikant und bedeutsam mit der Aufgabenübernahme des Vaters im Haushalt und bei der funktionalen sowie bei der zielgerichteten Kindererziehung. Das Engagement des Vaters hing wiederum signifikant mit dem Wohlbefinden der Mutter zusammen, allerdings bewegten sich die Korrelationskoeffizienten hier größtenteils in einem eher niedrigen Bereich. Die Teilnahme des Vaters an Aktivitäten der zielgerichteten Kindererziehung hing außerdem mit seiner Lebenszufriedenheit zusammen $(r=.176, p<.001)$, welche ihrerseits unabhängig von der Berufstätigkeit der Partnerin war $(r=-.010, p=.819)$ (nicht in der Tabelle).

Tabelle 3: Produkt-Moment-Korrelationen zwischen Umfang der Berufstätigkeit und Wohlbefinden der Mutter sowie Aufgabenübernahme des Vaters

\begin{tabular}{|c|c|c|c|c|c|}
\hline & 1 & 2 & 3 & 4 & 5 \\
\hline 1. Umfang Berufstätigkeit & - & & & & \\
\hline 2. Lebenszufriedenheit & .089 & - & & & \\
\hline 3. Stimmungsniveau & .100 & $.707^{\star}$ & - & & \\
\hline 4. Seelische Gesundheit & $.137^{*}$ & $.559^{*}$ & $.544^{*}$ & - & \\
\hline 5. Haushalt/ funkt. Kindererziehung & $.369^{*}$ & $.145^{\star}$ & $.167^{*}$ & $.159^{*}$ & - \\
\hline 6. Zielgerichtete Kindererziehung & $.147^{*}$ & $.218^{*}$ & $.150^{*}$ & .104 & $.602^{*}$ \\
\hline
\end{tabular}

Anmerkung: * nach Bonferroni-Korrektur $\left(p_{\text {krit }}=.0045\right)$ signifikant.

Die durchgeführte Mediatoranalyse ergab, dass die Partizipation des Vaters am Haushalt und an der funktionalen Kindererziehung den Zusammenhang zwischen der Berufstätigkeit und der seelischen Gesundheit der Mutter mediiert, wobei jedoch die korrigierte Varianzaufklärung im dritten Testschritt relativ schwach ausgeprägt ist (Abbildung 2). Es ergaben sich aufgrund der fehlenden Signifikanz der Zusammenhänge zwischen Berufstätigkeit und Lebenszufriedenheit bzw. Stimmungsniveau der Mutter keine weiteren Mediationseffekte. 
Abbildung 2: Aufgabenbeteiligung des Vaters als Mediatorvariable des Zusammenhangs zwischen Berufstätigkeit und seelischer Gesundheit der Mutter (Bootstrapping: indirekter Effekt $=0.0017, S E=0.0007, Z=2.4532$, $p=.014$; Bias-korrigiertes 95\%-Konfidenzintervall: 0.0005-0.0034)

Testschritt $2(\beta=.137 ; p=.002)$

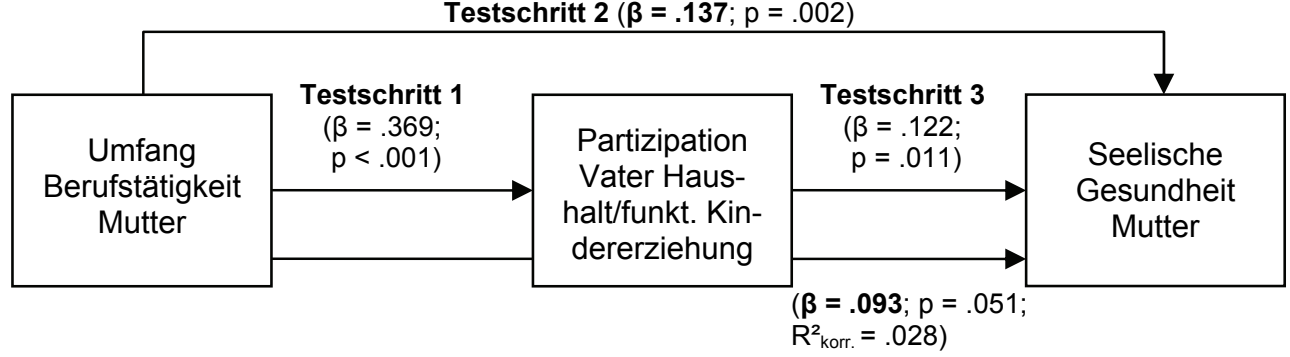

\subsection{Hypothese 2}

Die zur Prüfung von Hypothese 2 relevanten Korrelationen sind in Tabelle 4 abgetragen. Es wird erkennbar, dass ein höheres Wohlbefinden der Mutter durchgängig mit einem autoritativeren Erziehungsstil und zumindest teilweise mit einem weniger autoritären Erziehungsstil zusammenhing. Mit dem permissiven Erziehungsstil ergaben sich keine Zusammenhänge. Bezüglich der sozial-emotionalen Kompetenzen der Kinder zeigten sich signifikante und bedeutsame Zusammenhänge des Wohlbefindens der Mutter mit ihren Urteilen in den Skalen Peer Relations und Self-Management/Compliance. Zwischen der Lebenszufriedenheit der Mutter und der Skala Peerakzeptanz/globales Selbstwertgefühl zeigte sich ebenfalls ein signifikanter Zusammenhang, der zwar schwächer ausfiel, allerdings seine Signifikanz auch nach Bonferroni-Korrektur beibehielt. Der autoritative Erziehungsstil korrelierte signifikant und bedeutsam mit den Skalen Peer Relations und Self-Management/Compliance. Ansonsten zeigten sich nach Bonferroni-Korrektur keine signifikanten Zusammenhänge zwischen Erziehungsstil und sozial-emotionalen Kompetenzen.

Tabelle 4: Produkt-Moment-Korrelationen zwischen Wohlbefinden der Mutter, sozialemotionalen Kompetenzen des Kindes und Erziehungsstil

\begin{tabular}{|c|c|c|c|c|c|c|c|c|c|c|}
\hline & 1 & 2 & 3 & 4 & 5 & 6 & 7 & 8 & 9 & 10 \\
\hline 1. Lebenszufriedenheit & - & & & & & & & & & \\
\hline 2. Stimmungsniveau & $.707^{*}$ & - & & & & & & & & \\
\hline 3. Seelische Gesundheit & $.559^{*}$ & $.544^{\star}$ & - & & & & & & & \\
\hline 4. Peer Relations & $.293^{*}$ & $.252^{*}$ & $.256^{*}$ & - & & & & & & \\
\hline 5. Self-Management/ Compliance & $.326^{*}$ & $.248^{*}$ & $.301^{*}$ & $.683^{*}$ & - & & & & & \\
\hline 6. Selbstkonzept kognitive Kompetenz & .051 & -.008 & -.032 & .096 & .124 & - & & & & \\
\hline 7. Peerakzeptanz/ globales Selbstwertgefühl & $.142^{*}$ & .093 & .069 & $.257^{*}$ & $.193^{*}$ & $.465^{\star}$ & - & & & \\
\hline 8. Empathiefähigkeit & .057 & .060 & .000 & .104 & $.178^{*}$ & -.015 & -.009 & - & & \\
\hline 9. Autoritativer Erziehungsstil & $.202^{*}$ & $.169^{*}$ & $.265^{\star}$ & $.285^{\star}$ & $.298^{*}$ & -.082 & -.009 & .001 & - & \\
\hline 10. Autoritärer Erziehungsstil & -.090 & -.050 & $-.138^{*}$ & -.113 & -.095 & -.093 & -.102 & .044 & $-.135^{*}$ & - \\
\hline 11. Permissiver Erziehungsstil & -.069 & -.053 & -.032 & .020 & .016 & -.023 & .011 & -.031 & .110 & .097 \\
\hline
\end{tabular}


Die sich anschließenden Mediatoranalysen ergaben sechs partielle Mediationseffekte: Der autoritative Erziehungsstil vermittelte zwischen Lebenszufriedenheit bzw. Stimmungsniveau bzw. seelischer Gesundheit der Mutter und den Peer Relations bzw. Selbstmanagementfähigkeiten des Kindes, wobei sich die Effektstärken jeweils im mittleren Bereich bewegten (Abbildung 3). Die Ergebnisse des Bootstrappings zeigen, dass alle Mediationseffekte einen überzufälligen Beitrag zum jeweiligen Total-Effekt lieferten (Tabelle 5).

Abbildung 3: Autoritativer Erziehungsstil der Mutter als Mediatorvariable des Zusammenhangs zwischen Wohlbefinden der Mutter und sozialemotionalen Kompetenzen des Kindes

a

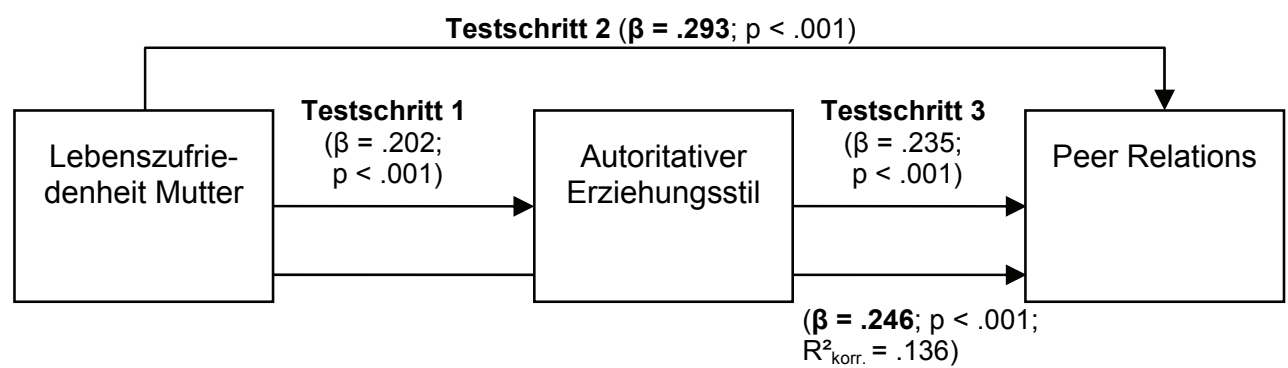

b

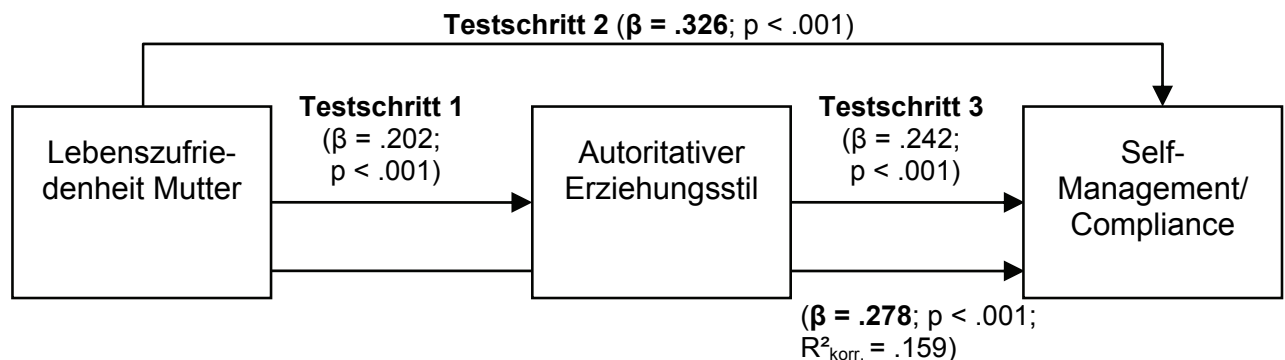

$\mathrm{C}$

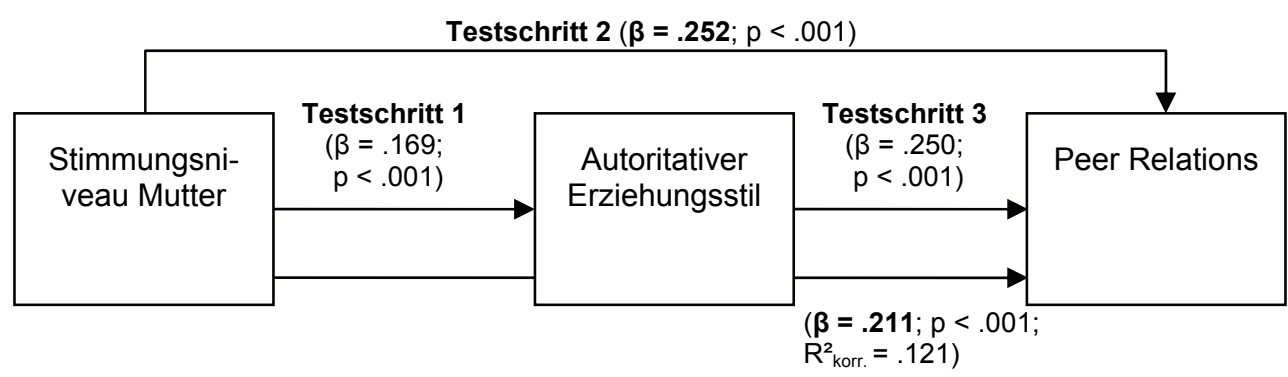


d

Testschritt $2(\beta=.248 ; p<.001)$

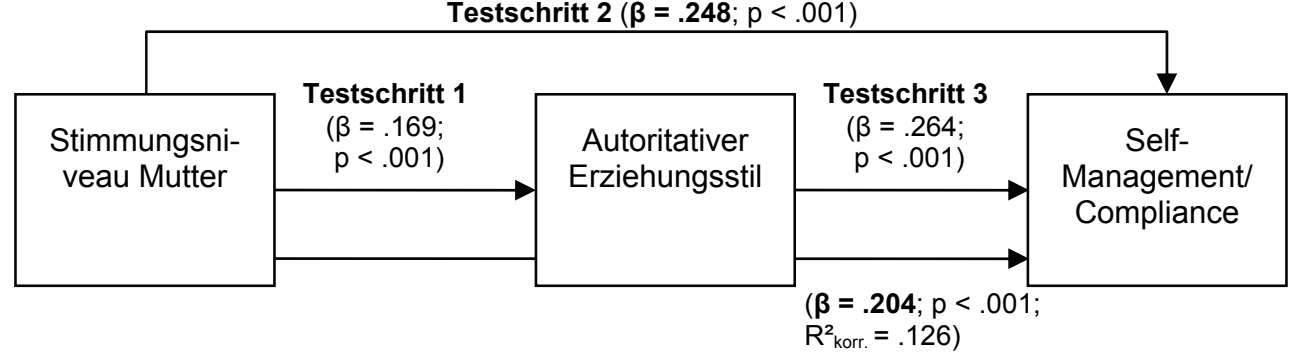

e

Testschritt $2(\beta=.256 ; p<.001)$

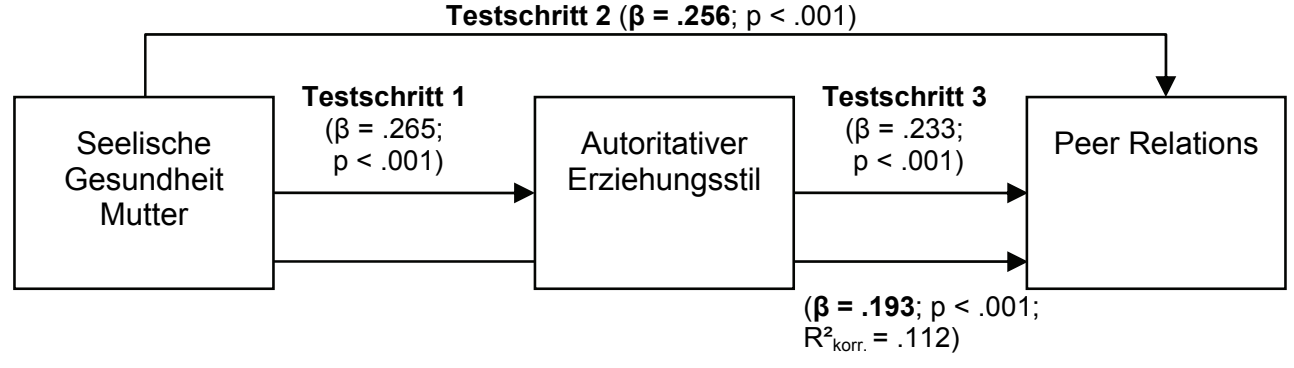

f

Testschritt $2(\beta=.301 ; p<.001)$

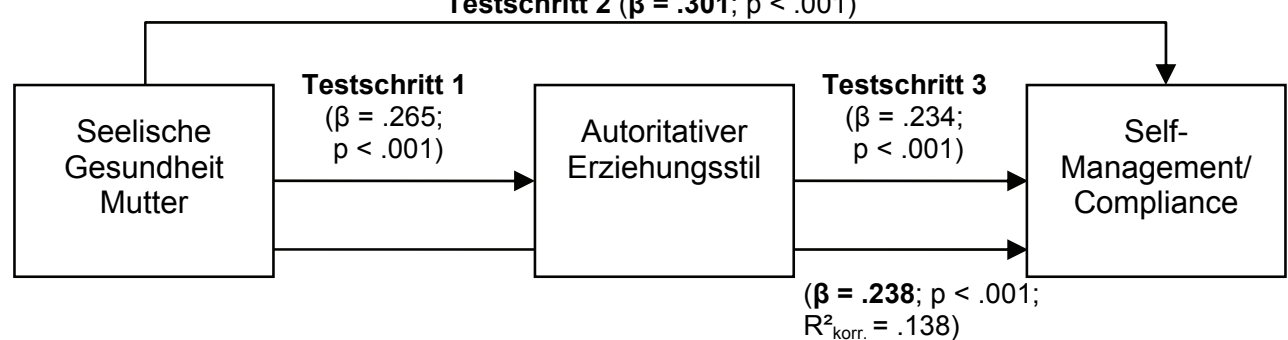

Tabelle 5: Mediationseffekte des autoritativen Erziehungsstils zwischen dem Wohlbefinden der Mutter und den sozial-emotionalen Kompetenzen des Kindes

\begin{tabular}{lcccccc}
\hline & $\begin{array}{c}\text { Indirekter } \\
\text { Effekt }\end{array}$ & SE & $\boldsymbol{Z}$ & $\boldsymbol{p}$ & \multicolumn{2}{c}{$\begin{array}{c}\text { Bootstrapping: BC 95\%-KI } \\
\text { Untere Grenze }\end{array}$} \\
\hline $\mathrm{a}$ & 0.0376 & 0.0106 & 3.5529 & $<.001$ & 0.0191 & 0.0637 \\
$\mathrm{~b}$ & 0.0407 & 0.0113 & 3.6090 & $<.001$ & 0.0210 & 0.0701 \\
$\mathrm{c}$ & 0.0293 & 0.0092 & 3.1662 & $<.001$ & 0.0126 & 0.0507 \\
$\mathrm{~d}$ & 0.0326 & 0.0101 & 3.2152 & $<.001$ & 0.0155 & 0.0582 \\
$\mathrm{e}$ & 0.0807 & 0.0198 & 4.0653 & $<.001$ & 0.0459 & 0.1291 \\
$\mathrm{f}$ & 0.0854 & 0.0208 & 4.1042 & $<.001$ & 0.0474 & 0.1397 \\
\hline
\end{tabular}

Anmerkungen: $\mathrm{BC}$ 95\%-KI = Bias-korrigiertes 95\%-Konfidenzintervall; 5.000 Bootstrap-Stichproben. 


\subsection{Hypothesen 3 und 4}

Zwischen Umfang der Berufstätigkeit der Mutter (Arbeitsstunden pro Woche) und den sozial-emotionalen Kompetenzen der Kinder ergaben sich keine signifikanten Zusammenhänge, auch nicht nach Berücksichtigung des Geschlechts. Wurde das Bildungsniveau in den Analysen berücksichtigt, zeigten sich nur vereinzelt Zusammenhänge, die nach Bonferroni-Korrektur allerdings keine Signifikanz mehr erreichten. Zwischen Berufstätigkeit und Erziehungsstil zeigten sich ebenfalls keine Zusammenhänge.

In einer pfadanalytischen Untersuchung konnte dementsprechend keine vollständige Bestätigung des Gesamtmodells gefunden werden, jedoch lieferten die Pfade Berufstätigkeit $\rightarrow$ Aufgabenbeteiligung (Haushalt) $\rightarrow$ seelische Gesundheit $\rightarrow$ Peer Relations bzw. Self-Management/Compliance (BC 95\%-KI: 0.0001-0.0010 bzw. 0.0002-0.0012), Berufstätigkeit $\rightarrow$ seelische Gesundheit $\rightarrow$ Peer Relations (BC 95\%-KI: 0.0001-0.0021) und Berufstätigkeit $\rightarrow$ Aufgabenbeteiligung (Haushalt) $\rightarrow$ seelische Gesundheit $\rightarrow$ autoritativer Erziehungsstil $\rightarrow$ Self-Management/Compliance (BC 95\%-KI: 0.0001-0.0004) einen signifikanten Beitrag zum jeweiligen Total-Effekt (s. Abbildung 4), der allerdings in beiden Fällen nicht signifikant wurde (Peer Relations: 0.0037, $p=.097$; Self-Management/ Compliance: $0.0022, p=.346$ ).

Im Rahmen explorativer Datenanalysen mittels multivariater Varianzanalysen wurde daher untersucht, ob die Kongruenz zwischen dem von der Mutter gewünschten und dem tatsächlichen Lebensmodell als Moderatorvariable für die Zusammenhänge zwischen Berufstätigkeit und sozial-emotionalen Kompetenzen, Wohlbefinden bzw. Erziehungsstil fungiert. Bezüglich der Selbstmanagementfähigkeiten der Kinder, des permissiven Erziehungsstils und sämtlicher Variablen des mütterlichen Wohlbefindens zeigte sich jedoch, dass der Haupteffekt der Kongruenz über den Interaktionseffekt beider Faktoren hinausgeht (vgl. Tabelle 6). Lediglich bei den Peer Relations zeigte sich das umgekehrte Bild. Bei nichtberufstätigen Müttern war die Ausprägung der Kongruenz irrelevant, während berufstätige Mütter, die Kongruenz empfanden, deutlich höhere Werte in den Peer Relations angaben als solche, die keine Kongruenz empfanden. Beim autoritativen und beim autoritären Erziehungsstil traten keinerlei Effekte auf (nicht in der Tabelle). 
Abbildung 4: Unstandardisierte Pfadkoeffizienten des postulierten Gesamtmodells $(* * p<.01, * * * p<.001$; fettgedruckte Pfeile bedeuten signifikante indirekte Effekte)

a (Total-Effekt: $0.0037, p=.097$ )

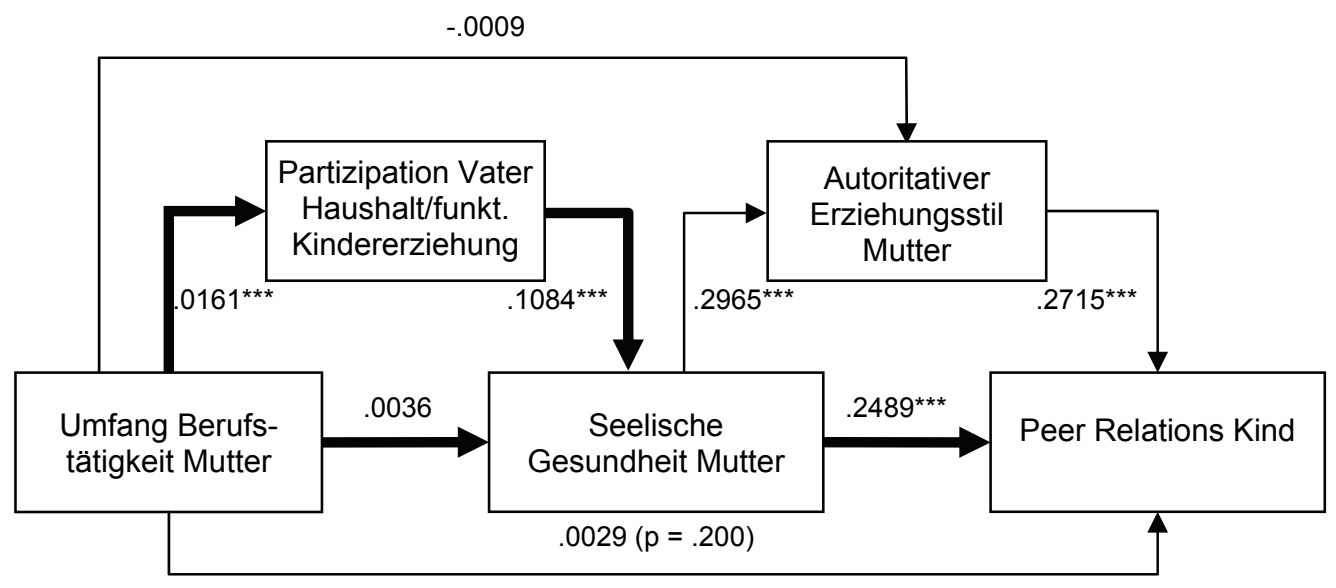

b (Total-Effekt: $0.0022, p=.346)$

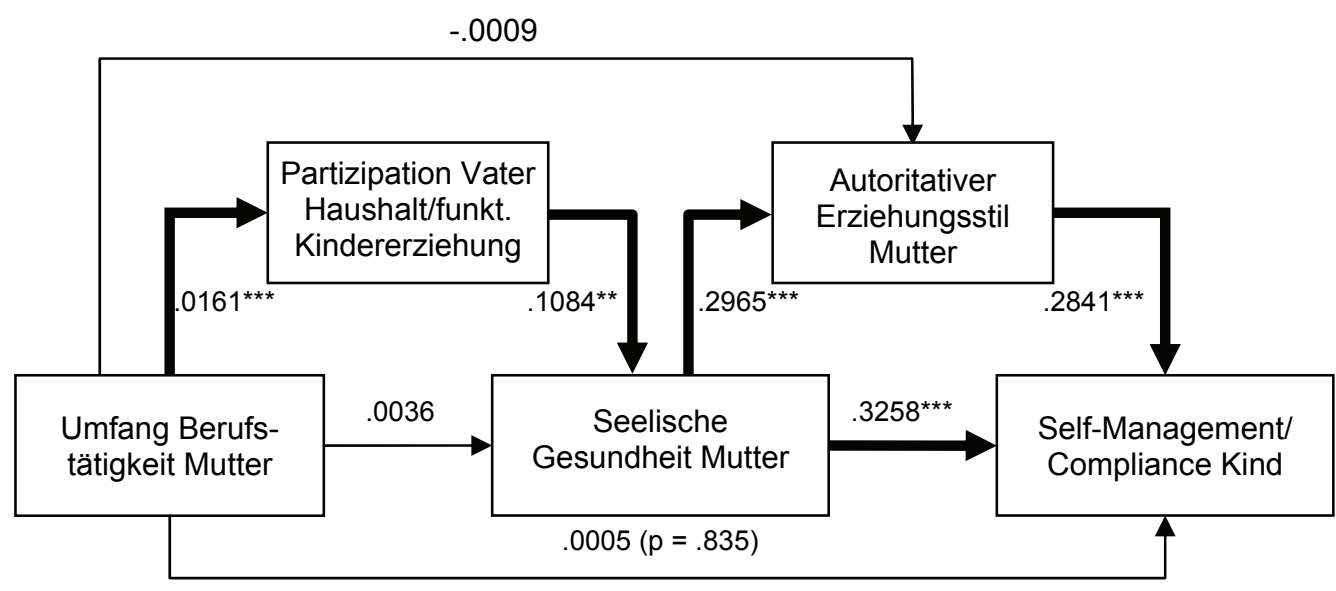


Tabelle 6: Ergebnisse der zweifaktoriellen MANOVA zu sozial-emotionalen Kompetenzen der Kinder sowie Erziehungsstil und Wohlbefinden der Mutter in Abhängigkeit vom Umfang der Berufstätigkeit und Kongruenz zwischen gewünschtem und tatsächlichem Umfang der Berufstätigkeit

\begin{tabular}{|c|c|c|c|c|}
\hline Skala & Faktoren & $F$ & $p$ & $\eta^{2}$ \\
\hline \multirow[t]{3}{*}{ Peer Relations } & Berufstätigkeit & 0.061 & .805 & .000 \\
\hline & Kongruenz & 1.565 & .211 & .003 \\
\hline & Berufstätigkeit*Kongruenz & 3.396 & .066 & .007 \\
\hline \multirow[t]{3}{*}{ Self-Management } & Berufstätigkeit & 0.064 & .801 & .000 \\
\hline & Kongruenz & 3.651 & .057 & .008 \\
\hline & BerufstätigkeitKongruenz & 0.016 & .899 & .000 \\
\hline Permissiver & Berufstätigkeit & 2.722 & .100 & .006 \\
\hline \multirow[t]{2}{*}{ Erziehungsstil } & Kongruenz & 6.698 & .010 & .014 \\
\hline & Berufstätigkeit*Kongruenz & 1.460 & .228 & .003 \\
\hline \multirow[t]{3}{*}{ Lebenszufriedenheit } & Berufstätigkeit & 1.159 & .282 & .002 \\
\hline & Kongruenz & 23.530 & .000 & .048 \\
\hline & Berufstätigkeit*Kongruenz & 6.056 & .014 & .013 \\
\hline \multirow[t]{3}{*}{ Stimmungsniveau } & Berufstätigkeit & 0.152 & .696 & .000 \\
\hline & Kongruenz & 12.557 & .000 & .026 \\
\hline & Berufstätigkeit*Kongruenz & 1.691 & .194 & .004 \\
\hline \multirow[t]{3}{*}{ Seelische Gesundheit } & Berufstätigkeit & 2.712 & .100 & .006 \\
\hline & Kongruenz & 3.863 & .050 & .008 \\
\hline & Berufstätigkeit*Kongruenz & 2.738 & .099 & .006 \\
\hline
\end{tabular}

Anmerkung: Da sich bezüglich autoritativem und autoritärem Erziehungsstil keinerlei Effekte ergaben, wurden beide Variablen nicht in die Tabelle aufgenommen.

Wurde im Gesamtmodell mit der abhängigen Variable Self-Management/Compliance die Kongruenz statt des Umfangs der Berufstätigkeit eingesetzt, wurden fünf der sieben Mediationspfade signifikant (s. Tabelle 7 und Abbildung 5). Der direkte Effekt der Kongruenz war stärker als derjenige der Berufstätigkeit und verpasste nur knapp das Signifikanzniveau. Der Total-Effekt wurde nun signifikant $(0.1324, p=.020)$.

Tabelle 7: Signifikante Mediationseffekte im modifizierten Gesamtmodell

\begin{tabular}{lllll}
\hline Mediationspfad & Indirekter Effekt & SE & \multicolumn{2}{c}{ Bootstrapping: BC 95\%-KI } \\
& & & Untere Grenze & Obere Grenze \\
\hline $\mathrm{K} \rightarrow \mathrm{A} \rightarrow \mathrm{G} \rightarrow \mathrm{S}$ & 0.0043 & 0.0025 & 0.0009 & 0.0113 \\
$\mathrm{~K} \rightarrow \mathrm{A} \rightarrow \mathrm{E} \rightarrow \mathrm{S}$ & 0.0024 & 0.0018 & 0.0002 & 0.0079 \\
$\mathrm{~K} \rightarrow \mathrm{A} \rightarrow \mathrm{G} \rightarrow \mathrm{E} \rightarrow \mathrm{S}$ & 0.0011 & 0.0007 & 0.0002 & 0.0032 \\
$\mathrm{~K} \rightarrow \mathrm{G} \rightarrow \mathrm{S}$ & 0.0297 & 0.0169 & 0.0027 & 0.0701 \\
$\mathrm{~K} \rightarrow \mathrm{G} \rightarrow \mathrm{E} \rightarrow \mathrm{S}$ & 0.0075 & 0.0043 & 0.0011 & 0.0185 \\
\hline
\end{tabular}

Anmerkungen: $\mathrm{K}=$ Kongruenz, $\mathrm{A}=$ Aufgabenteilung, $\mathrm{G}=$ seelische Gesundheit, $\mathrm{E}=$ autoritativer Erziehungsstil, $\mathrm{S}=$ Self-Management/Compliance; BC 95\%-KI = Bias-korrigiertes 95\%-Konfidenzintervall; 5.000 Bootstrap-Stichproben. 
Abbildung 5: Unstandardisierte Pfadkoeffizienten des modifizierten Gesamtmodells $\left({ }^{*} p<.05, * * p<.01, * * * p<.001\right.$; fettgedruckte Pfeile $=$ signifikante indirekte Effekte; graue Pfeile $=$ Zusammenhänge, die nicht postuliert wurden, aber signifikant wurden bzw. Bestandteil eines indirekten Effekts waren; Total-Effekt: 0.1324, $p=$ $.020)$

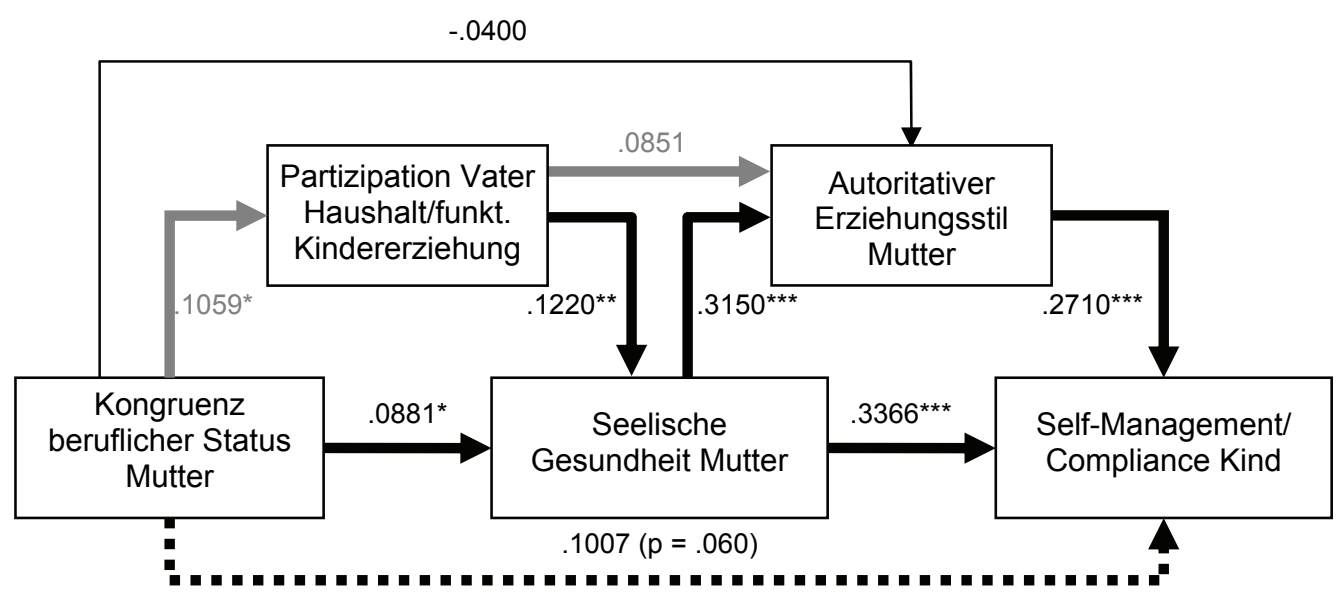

Der signifikante Zusammenhang zwischen Kongruenz und väterlicher Partizipation erscheint erklärungsbedürftig, da Kongruenz auch bei einer Frau, die sich aus freien Stücken ausschließlich über ihre Rolle als Hausfrau und Mutter definiert, vorliegen kann und in diesem Fall kein Grund besteht, von einer höheren Partizipation des Vaters an haushalts- und kindbezogenen Aufgaben auszugehen. Möglicherweise lässt sich dieses Ergebnis jedoch dadurch erklären, dass in der vorliegenden Stichprobe berufstätige Frauen mit Kongruenz nichtberufstätigen Frauen mit Kongruenz zahlenmäßig deutlich überlegen waren $(54.9 \%$ vs. $10.2 \%$ der Stichprobe), während bei den Frauen ohne Kongruenz die Nichtberufstätigen in der Überzahl waren $\left(19.7 \%\right.$ vs. $\left.15.2 \% ; \chi_{(1)}^{2}=84.979, p<.001\right)$ und signifikante Zusammenhänge zwischen mütterlicher Berufstätigkeit und Aufgabenübernahme des Partners bestanden.

\section{Diskussion}

Gegenstand der Studie war die Überprüfung eines Modells, das Zusammenhänge zwischen mütterlicher Berufstätigkeit, väterlicher Beteiligung an Haushalts- und Kindererziehungsaufgaben, Wohlbefinden der Mutter, Erziehungsstil sowie den sozial-emotionalen Kompetenzen des Kindes annimmt (s. Abbildung 1). Zu diesem Zweck wurden Daten von insgesamt 985 vollständigen Familien mit Kindern der 3., 4. und 6. Klassen ausgewertet. Die postulierten Zusammenhänge konnten zum Teil bestätigt werden. Für das Wohlbefinden der Mutter und die sozial-emotionalen Kompetenzen des Kindes scheint der Kongruenz zwischen gewünschtem und tatsächlichem Erwerbstätigkeitsumfang eine größere Bedeutung zuzukommen als der Berufstätigkeit an sich. 


\subsection{Zusammenfassung und Interpretation der Ergebnisse}

Die Auswertung der Daten ergab nur eine eingeschränkte Bestätigung von Hypothese 1. Der Umfang der mütterlichen Berufstätigkeit, das Wohlbefinden der Mutter sowie die Aufgabenübernahme des Vaters im Haushalt und bei der Kindererziehung korrelierten positiv miteinander, jedoch waren die Zusammenhänge zwischen dem Umfang der Berufstätigkeit und Wohlbefinden eher schwach ausgeprägt und wurden nur für die seelische Gesundheit signifikant. Dieser Zusammenhang wurde durch die Partizipation des Vaters an familiären Aufgaben mediiert, was die Annahme stützt, dass engagierte Partner berufstätige Mütter von ihrer Doppelrolle entlasten und so für ein höheres Wohlbefinden sorgen können (Hoffman/Youngblade 1999). Aufgrund der schwachen Zusammenhänge kann nicht von einer uneingeschränkten Stützung der Bereicherungshypothese und der Ergebnisse aus vorangegangenen Studien (Repetti et al. 1989; Hoffman/Youngblade 1999; Klumb/Lampert 2004; Richter et al. 2007; Buehler/O’Brien 2011) gesprochen werden.

Vielmehr scheint die Passung zwischen gewünschter und tatsächlicher Berufstätigkeit von Bedeutung für das Wohlbefinden der Mutter zu sein. Wenn Kongruenz gegeben war, gaben die Mütter eine signifikant höhere Lebenszufriedenheit, seelische Gesundheit und ein besseres Stimmungsniveau an, als wenn sie lieber weniger oder mehr arbeiten wollten. Dies steht in Einklang mit den Befunden von Berger (2009), die in ihrer Studie mit 5.706 Müttern zu dem Ergebnis kam, dass Mütter, die gerne mehr arbeiten wollten, dies aber aufgrund familiärer Einschränkungen nicht konnten, eine geringere Lebenszufriedenheit aufwiesen als Vollzeit berufstätige Mütter. Die entscheidenden Faktoren waren dabei entgangene monetäre Vorteile, ein geringeres soziales Netzwerk, fehlende Selbstwertbestätigung, fehlende Ablenkung von häuslichen Aufgaben, sinkende Karrierechancen und ein geringes, wenig befriedigendes Niveau von Teilzeittätigkeiten. In eine ähnliche Richtung weisen die Ergebnisse einer weiteren Untersuchung mit 863 Elternpaaren von RöhrSendlmeier/Bergold/Krüger (2013), nach denen traditionell eingestellte berufstätige Mütter mehr Schuldgefühle gegenüber ihren Kindern angaben als egalitär eingestellte berufstätige Mütter. Die Ergebnisse scheinen somit eher für das Person-Environment-Fit-Modell (Klumb/Lampert 2004) zu sprechen, nach dem Gesundheit und Wohlbefinden dann am höchsten ausgeprägt sind, wenn die ausgeübten Rollen den eigenen Wünschen, Vorstellungen und Fähigkeiten entsprechen. Demnach sind Rollen (z.B. als berufstätige Mutter oder als Hausfrau und Mutter) nicht an sich dem Wohlbefinden abträglich oder förderlich, sondern immer nur in Verbindung mit den Wünschen, Erwartungen und Fähigkeiten einer Person.

Hypothese 2 konnte in weiten Teilen bestätigt werden. Je höher das Wohlbefinden der Mütter war, desto häufiger gaben sie an, den autoritativen Erziehungsstil anzuwenden. Zudem war der autoritäre Stil umso schwächer ausgeprägt, je höher die Mütter ihre seelische Gesundheit einschätzten. Der autoritative Erziehungsstil korrelierte wiederum in erwarteter Richtung und signifikant mit den Peer Relations und den Selbstmanagementfähigkeiten der Kinder. Die Skalen Peer Relations und Self-Management/Compliance korrelierten außerdem signifikant positiv mit allen drei Skalen zum Wohlbefinden der Mutter, die Skala Peerakzeptanz/globales Selbstwertgefühl zumindest mit der Lebenszufriedenheit. Der autoritative Erziehungsstil vermittelte den positiven Zusammenhang zwischen mütterlichem Wohlbefinden und den Peer Relations bzw. Selbstmanagement- 
fähigkeiten des Kindes partiell. Diese Befunde stehen in Einklang mit Studienergebnissen, die einen Zusammenhang zwischen positivem Erziehungsstil und sozial-emotionalen Kompetenzen der Kinder zeigen konnten (Deković/Janssens 1992; Hoffman/Youngblade 1999; Franiek/Reichle 2007; vgl. auch Reichle/Gloger-Tippelt 2007), und untermauern die Annahme, dass der positive Effekt eines hohen elterlichen Wohlbefindens auf die sozial-emotionalen Kompetenzen des Kindes über den Erziehungsstil vermittelt wird (Hoffman/Youngblade 1999).

Ein direkter Zusammenhang zwischen dem Umfang der mütterlichen Berufstätigkeit und sozial-emotionalen Kompetenzen der Kinder konnte nicht gefunden werden, so dass die Hypothesen 3 und 4 nicht nach der causal steps - Methode von Baron/Kenny (1986) überprüft werden konnten. Befunde aus dem anglo-amerikanischen Raum, nach denen die mütterliche Berufstätigkeit an sich eine positive Einflussgröße darstellt (Hoffman/Youngblade 1999; McMunn et al. 2011), konnten somit nicht bestätigt werden. In den MetaAnalysen von Goldberg et al. (2008) und Lucas-Thompson et al. (2010) waren mit dem Familienstand, dem Alter der Kinder und besonders dem sozioökonomischen Status wichtige Moderatorvariablen identifiziert worden, die auf diesen Zusammenhang einwirken. Das Bildungsniveau hatte in der vorliegenden Studie jedoch keinen Einfluss. Auch hinsichtlich des Erziehungsstils fanden sich keine Effekte der Berufstätigkeit, was Befunden aus englischsprachigen Studien (Hoffman/Youngblade 1999; Raver 2003; Gottfried/Gottfried 2006; Buehler/O'Brien 2011) ebenfalls widerspricht. Möglicherweise müssen hier kulturspezifische Einflüsse berücksichtigt werden.

In der vorliegenden Studie spielte für die sozial-emotionalen Fähigkeiten der Kinder die Kongruenz zwischen gewünschtem und realisiertem Beschäftigungsstatus eine wichtigere Rolle als der Umfang der Berufstätigkeit allein. Lag Kongruenz vor, erzielten die Kinder höhere Werte auf der Skala Self-Management/Compliance. Bezüglich des Zusammenhangs zwischen mütterlicher Berufstätigkeit und Peer Relations wirkte die Kongruenz als Moderatorvariable, wobei allerdings das Signifikanzniveau knapp verpasst wurde. Hierbei muss außerdem berücksichtigt werden, dass es sich um Einschätzungen der Mütter zu den sozial-emotionalen Kompetenzen ihrer Kinder handelt. In den Angaben, die die Kinder machten, zeigten sich keine entsprechenden Unterschiede. Möglicherweise liegt eine Verzerrung im Urteil zufriedenerer Mütter vor. Gottfried/Gottfried (2006) konnten jedoch ebenfalls zeigen, dass Kinder von Müttern, die mit ihrer jeweiligen Rolle zufrieden waren, eine höhere Leistungsmotivation besaßen und weniger Verhaltensprobleme zeigten als Kinder unzufriedener Mütter. In einer Untersuchung von Hart/Kelley (2006) berichteten Eltern umso mehr internalisierende (z.B. Ängste, Sorgen, Traurigkeit) und externalisierende (z.B. Aggressivität) Verhaltensweisen ihrer Kinder, je stärker sie einen Konflikt zwischen Beruf und Familie und Stress in ihrer Elternrolle erlebten. Zudem muss beachtet werden, dass die Kinder in der vorliegenden Untersuchung andere Skalen bearbeiteten als die Mütter. Möglicherweise hätten sich Unterschiede gezeigt, wenn auch die Kinder selber ihre Peer-Beziehungen und ihre Selbstmanagementfähigkeiten eingeschätzt hätten. Die Kongruenzhypothese ebenfalls stützende Ergebnisse konnten in Analysen der familiären Rahmenbedingungen bei 335 Familien für den Bereich der kognitiven Kompetenzen mittels Automatic Interaction Detector und Konfigurationsfrequenzanalyse gefunden werden (Martin 2009; Rahmann 2009): Mütter mit höherer Bildung und entsprechenden Berufen sowie akademisch nicht gebildete Mütter, die nicht berufstätig waren und ihre 
Rolle primär als Hausfrau und Mutter sahen, hatten signifikant eher überdurchschnittlich begabte Kinder als Mütter mit akademischer Bildung in nicht-akademischen Berufen und Mütter, die sich als arbeitslos bezeichneten. Die Kongruenz des beruflichen Status war nach der Arbeitsgeschwindigkeit bzw. dem Verhalten während der Testsituation der zweitstärkste Prädiktor für die verbale (Varianzaufklärung: 6.02\%) bzw. für die nonverbale (Varianzaufklärung: $7.02 \%)$ Intelligenz der Kinder.

Aufgrund der bedeutsamen Rolle der Kongruenz sowohl für das Wohlbefinden der Mütter als auch für die sozial-emotionale Entwicklung der Kinder wurde ein Alternativmodell zum ursprünglichen Modell vorgeschlagen, das an die Stelle des Umfangs der Berufstätigkeit die Kongruenz zwischen gewünschtem und realisiertem Beschäftigungsstatus der Mutter setzt. Die pfadanalytische Überprüfung dieses Modells erbrachte im Vergleich zum ursprünglichen Modell mehr signifikante Pfadkoeffizienten, mehr signifikante vermittelnde Effekte und einen signifikanten Total-Effekt. In zukünftigen Studien zum Zusammenhang zwischen Berufstätigkeit, Wohlbefinden und Auswirkungen auf Kindvariablen könnte es daher lohnend sein, weniger die Berufstätigkeit an sich, als vielmehr die Passung zwischen gewünschtem und realisiertem Lebensmodell als entscheidende Variable in Betracht zu ziehen.

\subsection{Limitationen und Ausblick}

Es ist auf einige Einschränkungen hinzuweisen, denen diese Studie unterliegt: Die Ergebnisse wurden aus einem Querschnitts- bzw. Ex-post-facto-Design gewonnen, welches keine gesicherten Aussagen über Kausalitätsbeziehungen erlaubt. Z.B. ist durchaus denkbar, dass sozial-emotionale Kompetenzen des Kindes auf den Erziehungsstil und die Zufriedenheit der Eltern einwirken bzw. dass reziproke Beziehungen bestehen. Gleiches gilt für die - wenn auch hier nur schwachen - Zusammenhänge zwischen Berufstätigkeit und Wohlbefinden. Zwar wurde versucht, durch Mediatoranalysen Hinweise auf kausale Wirkungen zu erhalten; diese sollten im Idealfall jedoch z.B. in prospektiven Längsschnittstudien untersucht werden. Eine Alternativerklärung für die gefundenen Ergebnisse könnten z.B. auch Moderatoreffekte der hier als Mediatoren untersuchten Variablen sein.

Am ausschließlichen Einsatz der Fragebogenmethode ist zu kritisieren, dass Fragebogendaten keine Informationen über tatsächlich gezeigtes Verhalten liefern. Bezüglich der sozial-emotionalen Kompetenzen hätten auch Daten aus Verhaltensbeobachtungen gesammelt werden können. Dies wäre besonders aufschlussreich gewesen, da sich in den Auskünften der Mütter postulierte Effekte zeigten, nicht jedoch in den Selbstauskünften der Kinder. Hier kann auch nicht ausgeschlossen werden, dass zumindest ein gewisser Teil der Zusammenhänge auf die gemeinsame Methodenvarianz zurückgeht. Die Aufgabenpartizipation der Väter wurde nur von diesen erfragt. Es wäre optimal gewesen, auch die Mütter dazu zu befragen und anschließend beide Angaben zu vergleichen und ggf. einen Mittelwert zu bilden. Die Gefahr von Verfälschungstendenzen ist trotz der Anonymitätszusicherung gegeben.

Da die Erhebung auf freiwilliger Basis erfolgte, können systematische Selbstselektionseffekte nicht ausgeschlossen werden. Die Stichprobe umfasste zwar Familien aus urbanen, suburbanen und ländlichen Gebieten, dennoch waren Eltern mit akademischer Ausbildung überrepräsentiert, welche in der Regel über höhere ökonomische, kulturelle 
und soziale Ressourcen verfügen, die der Entwicklung der Kinder zuträglich sind (Bos/ Stubbe/Buddeberg 2010).

Dennoch weisen die Ergebnisse darauf hin, dass Eltern die Möglichkeit gegeben werden sollte, ihr favorisiertes Lebensmodell zu realisieren, da sich dies positiv auf Wohlbefinden und Zufriedenheit und damit auch auf die sozial-emotionale Entwicklung der Kinder auswirken kann. Dass hier noch großer Handlungsbedarf besteht, zeigen aktuelle Umfrageergebnisse: In einer repräsentativen Befragung des Forsa-Instituts (2008) sagten $44 \%$ der Mütter, dass sie gerne mehr arbeiten würden, die aktuelle Kinderbetreuungssituation dies aber nicht zulasse; in der Untersuchung von Berger (2009) wollten 57\% der nichtberufstätigen Mütter gerne einer Erwerbstätigkeit nachgehen, sahen sich jedoch durch familiäre Aufgaben gebunden. Die Schaffung zusätzlicher Kinderbetreuungsplätze, flexiblerer Arbeitszeiten und -plätze und anspruchsvoller Teilzeittätigkeiten scheint dringend geboten, möchte man Müttern, die gerne oder in größerem Umfang berufstätig wären, eine entsprechende Erwerbstätigkeit auch tatsächlich ermöglichen.

\section{Literatur}

Abramovitch, R. \& Johnson, L. C. (1992). Children's perceptions of parental work. Canadian Journal of Behavioral Science, 24, S. 319-332.

Almeida, D. M., Maggs, J. L. \& Galambos, N. L. (1993). Wives' employment hours and spousal participation in family work. Journal of Family Psychology, 7, S. 233-244.

Armistead, L., Wierson, M. \& Forehand, R. (1990). Adolescents and maternal employment: Is it harmful for a young adolescent to have an employed mother? Journal of Early Adolescence, 10, S. 260-278.

Asendorpf, J. B. \& Aken, M. A. G. von (1993). Self-Perception Profile for Children-deutsche Fassung (SPPC-D). Berlin: Humboldt-Universität, Institut für Psychologie.

Baron, R. M. \& Kenny, D. A. (1986). The moderator-mediator variable distinction in social psychological research: Conceptual, strategic, and statistical considerations. Journal of Personality and Social Psychology, 51, S. 1173-1182.

Baumrind, D. (1966). Effects of authoritative parental control on child behavior. Child Development, 37, S. 887-907.

Becker, P. (1989). Trierer Persönlichkeitsfragebogen (TPF). Göttingen: Hogrefe.

Berger, E. M. (2009). Maternal employment and happiness: The effect of non-participation and parttime employment on mothers' life satisfaction. Berlin: Deutsches Institut für Wirtschaftsforschung (Discussion Paper 890).

Berger, H. (2011). Berufstätigkeit, Zufriedenheit und Leistungsmotivation: Ein Vergleich von Eltern mit kinderlosen Erwerbstätigen. Bonn: Universität Bonn, Institut für Psychologie (Diplomarbeit).

Bos, W., Stubbe, T. C. \& Buddeberg, M. (2010). Gibt es eine armutsbedingte Bildungsbenachteiligung? Die Operationalisierung verschiedener Indikatoren der sozialen Herkunft in der empirischen Bildungsforschung. In: Rost, D. H. (Hrsg.), Intelligenz, Hochbegabung, Vorschulerziehung, Bildungsbenachteiligung. Münster: Waxmann, S. 165-208.

Buehler, C. \& O'Brien, M. (2011). Mothers' part-time employment: Associations with mother and family well-being. Journal of Family Psychology. doi: 10.1037/ a0025993.

Bundesministerium für Familie, Senioren, Frauen und Jugend (BMFSFJ) (Hrsg.) (2010). Perspektive Wiedereinstieg. Ziele, Motive und Erfahrungen von Frauen vor, während und nach dem beruflichen Wiedereinstieg. http://www.bmfsfj.de/RedaktionBMFSFJ/Broschuerenstelle/Pdf-Anlagen/PerspektiveWiedereinstieg-Ziele-Motive-Erfahrungen,property $=$ pdf,bereich $=$ bmfsfj, sprache $=$ de,rwb=true.pdf [Stand: 2011-11-14]. 
Crouter, A. C. \& Crowley, M. S. (1990). School-age children's time alone with fathers in single- and dual-earner families: Implications for the father-child relationship. Journal of Early Adolescence, 10, S. 296-312.

Dalbert, C. (1992). Subjektives Wohlbefinden junger Erwachsener: Theoretische und empirische Analysen der Struktur und Stabilität. Zeitschrift für Differentielle und Diagnostische Psychologie, 13, S. 207-220.

Deković, M. \& Janssens, J. M. A. M. (1992). Parents' child-rearing style and child's sociometric status. Developmental Psychology, 28, S. 925-932.

Forsa-Institut (2008). Lebensgefühl von Eltern. Repräsentativbefragung für Gruner + Jahr AG \& Co $K G$, Redaktion ELTERN. Tabellenband. http://c1.eltern.de/pdf/Tab-Lebensgefuehl-von-Eltern P8490_v2.pdf [Stand: 2011-11-25].

Franiek, S. \& Reichle, B. (2007). Elterliches Erziehungsverhalten und Sozialverhalten im Grundschulalter. Kindheit und Entwicklung, 16, S. 240-249.

Fthenakis, W. E. (1999). Vaterschaft - gestern und heute. In: LBS-Initiative Junge Familie (Hrsg.), Engagierte Vaterschaft. Die sanfte Revolution in der Familie. Opladen: Leske + Budrich, S. 15-40.

GEOlino \& Kinderhilfswerk der Vereinten Nationen (UNICEF) (2010). GEOlino-Kinderwertemonitor 2010. http://www.unicef.de/fileadmin/content_media/presse/Ergebnisse_Kinderwerte_Monitor_2010_ Praesentation_gesamt2.6.pdf [Stand: 2011-11-15].

Goldberg, W. A., Prause, J., Lucas-Thompson, R. G. \& Himsel, A. (2008). Maternal employment and children's achievement in context: A meta-analysis of four decades of research. Psychological Bulletin, 134, S. 77-108.

Gottfried, A. E. \& Gottfried, A. W. (2006). A long-term investigation of the role of maternal and dualearner employment in children's development: The Fullerton Longitudinal Study. American Behavioral Scientist, 49, S. 1310-1327.

Gove, W. R. (1984). Gender differences in mental and physical illness: The effect of fixed roles and nurturant roles. Social Science \& Medicine, 19, S. 77-91.

Hart, S. M. \& Kelley, M. L. (2006). Fathers' and mothers' work and family issues as related to internalizing and externalizing behaviour of children attending day care. Journal of Family Issues, 27, S. 252-270.

Hayes, A. F. (2012). An analytical primer and computational tool for observed variable mediation, moderation, and conditional process modeling (submitted for publication). http://www.afhayes. com/public/process.pdf [Stand: 2012-04-07].

Hoffman, L. W. (1989). Effects of maternal employment in the two-parent family. American Psychologist, 44, S. 283-292.

Hoffman, L. W. \& Youngblade, L. M. (1999). Mothers at work. Effects on children's well-being. New York, NY: Cambridge University Press.

Hölling, H. \& Schlack, R. (2008). Psychosoziale Risiko- und Schutzfaktoren für die psychische Gesundheit im Kindes- und Jugendalter - Ergebnisse aus dem Kinder- und Jugendgesundheitssurvey (KiGGS). Gesundheitswesen, 70, S. 154-163.

Hurrelmann, K. \& Andresen, S. (2010). Kinder in Deutschland 2010. 2. World Vision Kinderstudie. Frankfurt am Main: Fischer.

Jahoda, M. (1981). Work, employment und unemployment. Values, theories, and approaches in social research. American Psychologist, 36, S. 184-191.

Jaursch, S. \& Lösel, F. (2011). Mütterliche Berufstätigkeit und kindliches Sozialverhalten. Kindheit und Entwicklung, 20, S. 164-172.

Klumb, P. L. \& Lampert, T. (2004). Women, work, and well-being 1950-2000: a review and methodological critique. Social Science \& Medicine, 58, S. 1007-1024.

Lehr, U. (1979). Ist Frauenarbeit schädlich? Im Spannungsfeld von Familie und Beruf. Zürich: Edition Interfrom.

Lucas-Thompson, R. G., Goldberg, W. A. \& Prause, J. (2010). Maternal work early in the lives of children and its distal associations with achievement and behavior problems: A meta-analysis. Psychological Bulletin, 136, S. 915-942. 
Martin, D. (2009). Die Entwicklung von Grundschülern mit Verdacht auf Hochbegabung. Bonn: Universität Bonn, Institut für Psychologie (Diplomarbeit).

McMunn, A., Kelly, Y., Cable, N. \& Bartley, M. (2011). Maternal employment and child socioemotional behaviour in the UK: longitudinal evidence from the UK Millennium Cohort Study. Journal of Epidemiology and Community Health, doi: 10.1136/jech.2010.109553.

Merrell, K. W. \& Caldarella, P. (2008). Home \& Community Social Behavior Scales (HCSBS). East Peoria, IL: Versa Press.

Müller-Günther, M. (2010). Rahmenbedingungen des Lernens bei begabten Kindern. Bonn: Universität Bonn (Dissertation) http://hss.ulb.uni-bonn.de/2010/2274/ 2274.pdf [Stand: 2011-09-16].

Pache, M. (2009). Determinanten der Leistungsmotivation deutsch- und türkischstämmiger Schüler. Bonn: Universität Bonn, Institut für Psychologie (Diplomarbeit).

Paul, K. \& Moser, K. (2001). Negatives psychisches Befinden als Wirkung und als Ursache von Arbeitslosigkeit: Ergebnisse einer Meta-Analyse. In: Zempel, J., Bacher, J. \& Moser, K. (Hrsg.), Erwerbslosigkeit. Opladen: Leske + Budrich, S. 83-110.

Preacher, K. J. \& Hayes, A. F. (2008). Asymptotic and resampling strategies for assessing and comparing indirect effects in multiple mediator models. Behavior Research Methods, 40, S. 879-891.

Rahmann, J. (2009). Entwicklung, Verhalten und kognitive Leistungsfähigkeit von vermeintlich unterforderten Kindern und Jugendlichen - Eine Erhebung aus einer Beratungsstelle für Hochbegabte. Bonn: Universität Bonn, Institut für Psychologie (Diplomarbeit).

Raver, C. C. (2003). Does work pay psychologically as well as economically? The role of employment in predicting depressive symptoms and parenting among low-income families. Child Development, 74, S. 1720-1736.

Reichle, B. \& Gloger-Tippelt, G. (2007). Familiale Kontexte und sozial-emotionale Entwicklung. Kindheit und Entwicklung, 16, S. 199-208.

Repetti, R. L., Matthews, K. A. \& Waldron, I. (1989). Employment and women's health. Effects of paid employment on women's mental and physical health. American Psychologist, 44, S. 1394-1401.

Richter, R., Bergmann, R. L., Bergmann, K. E. \& Dudenhausen, J. W. (2007). Berufstätigkeit und Lebensqualität von Müttern zwei Jahre nach der Geburt des ersten Kindes. Gesundheitswesen, 69, S. 448-456.

Röhr-Sendlmeier, U. M. (2009). Berufstätige Mütter und die Schulleistungen ihrer Kinder. Bildung und Erziehung, 62, S. 225-242.

Röhr-Sendlmeier, U. M. \& Bergold, S. (2012). Die Rolle des Vaters bei berufstätiger Mutter - Aufgabenbeteiligung, Lebenszufriedenheit und Schulleistung der Kinder. Zeitschrift für Familienforschung/Journal of Family Research, 24, S. 3-26.

Röhr-Sendlmeier, U. M., Bergold, S. \& Krüger, M. (2013). Eltern im Konflikt zwischen Familie und Beruf - elterliche Schuldgefühle und ihr Zusammenhang mit sozial-emotionalen und schulischen Kompetenzen der Kinder (in Begutachtung).

Röhr-Sendlmeier, U. M. \& Kröger, M. (2011). Die Bedeutung der mütterlichen Berufstätigkeit für Leistungsmotivation und Berufswahlreife von Jugendlichen. Bildung und Erziehung, 64, S. 213-238.

Röhr-Sendlmeier, U. M., Schäfer, B. \& Vonderbank, C. (2009). Kindliche Rollenvorstellungen vom Erwachsensein im Kontext gewandelter Geschlechtsstereotype. Kinder- und Jugendschutz in Wissenschaft und Praxis, 54, S. 107-111.

Rutter, M. (1990). Commentary: Some focus and process considerations regarding effects of parental depression on children. Developmental Psychology, 26, S. 60-67.

Schnittert, C. (2011). Auswirkungen mütterlicher Berufstätigkeit auf den Schulerfolg von HauptschülerInnen. Bonn: Universität Bonn, Institut für Psychologie (Diplomarbeit).

Schwartzberg, N. S. \& Dytell, R. S. (1996). Dual earner families: The importance of work stress and family stress for psychological well-being. Journal of Occupational Health Psychology, 1, S. 211223.

Stadler, C., Janke, W. \& Schmeck, K. (2004). Inventar zur Erfassung von Impulsivität, Risikoverhalten und Empathie bei 9- bis 14-jährigen Kindern (IVE). Göttingen: Hogrefe. 
Statistisches Bundesamt (2011). Pressemitteilung Nr. 090 vom 08.03.2011. http://www.destatis.de/ jetspeed/portal/cms/Sites/destatis/Internet/DE/Presse/pm/2011/03/PD11_090_122.psml [Stand: 2011 -11-12].

Waldron, I., Weiss, C. C. \& Hughes, M. E. (1998). Interacting effects of multiple roles on women's health. Women and Health, 11, S. 79-98.

Wethington, E. \& Kessler, R. C. (1989). Employment, parental responsibility, and psychological distress. A longitudinal study of married women. Journal of Family Issues, 10, S. 527-546.

Wiese, B. S. (2004). Konflikte zwischen Beruf und Familie im Alltagserleben erwerbstätiger Paare. Querschnittliche und prozessuale Analysen. Zeitschrift für Sozialpsychologie, 35, S. 45-58.

Engereicht am/Submitted on: 24.01.2012

Angenommen am/Accepted on: 19.07.2012

Anschriften der Autorinnen und Autoren/Addresses of the authors:

Prof. Dr. Una Röhr-Sendlmeier (Korrespondenzautorin/corresponding author)

Dr. Sebastian Bergold

Andreas Jöris, Diplom-Psychologe

Anna Verena Cummings, Diplom-Psychologin

Karin Helm, Diplom-Psychologin

Eva Johannen, Diplom-Psychologin

Rheinische Friedrich-Wilhelms-Universität Bonn

Institut für Psychologie

Entwicklungspsychologie und Pädagogische Psychologie

Kaiser-Karl-Ring 9

53111 Bonn

Deutschland/Germany

E-Mail: roehr-sendlmeier@uni-bonn.de sbergold@uni-bonn.de ajoeris@uni-bonn.de 